\title{
Post-T Tauri Stars in the Nearest OB Association
}

\author{
Eric E. Mamajek, Michael R. Meyer, \& James Liebert \\ Steward Observatory, Dept. of Astronomy, University of Arizona, 933 N. Cherry Ave., Tucson, AZ 85721 \\ eem@as . arizona. edu
}

\begin{abstract}
We present results of a spectroscopic survey of X-ray- and proper motion-selected samples of late-type stars in the Lower Centaurus-Crux (LCC) and Upper Centaurus-Lupus (UCL) subgroups of the nearest $\mathrm{OB}$ association: Scorpius-Centaurus. The primary goals of the survey are to determine the star-formation history of the OB subgroups, and to assess the frequency of accreting stars in a sample dominated by "post-T Tauri" pre-main sequence (pre-MS) stars. We investigate two samples: (1) proper motion candidates from the ACT and TRC astrometric catalogs (Hoogerwerf 2000) with X-ray counterparts in the ROSAT All-Sky Survey (RASS) Bright Source Catalog, and (2) G and K-type stars in the Hipparcos catalog found to be candidate members by de Zeeuw et al. (1999). We obtained optical spectra of 130 candidates with the Siding Springs 2.3-m dual-beam spectrograph. Pre-MS stars were identified by (1) strong Li $\lambda 6707$ absorption, (2) subgiant surface gravities, (3) proper motions consistent with Sco-Cen membership, and (4) HR diagram positions consistent with being pre-MS. We find $93 \%$ of the RASS-ACT/TRC stars to be probable pre-MS members, compared to $73 \%$ of the Hipparcos candidates. We demonstrate that measuring the gravity-sensitive band-ratio of Sr II $\lambda 4077$ to Fe I $\lambda 4071$ is a valuable means of discriminating pre-MS and zero-age main sequence (ZAMS) stars. Using secular parallaxes, and Hipparcos, Tycho-2, and 2MASS photometry, we construct an HR diagram. Depending on the choice of published evolutionary tracks, we find the mean ages of the pre-MS populations to range between 17-23 Myr for LCC, and 15-22 Myr for UCL. Taking into account observational errors, it appears that $95 \%$ of the low-mass star-formation in each subgroup must have occurred in less than $8 \mathrm{Myr}$ (LCC) and $12 \mathrm{Myr}$ (UCL). Using the Bertelli et al. (1994) tracks, we find main sequence turn-off ages for Hipparcos B-type members to be $16 \pm 1 \mathrm{Myr}$ for LCC and $17 \pm 1 \mathrm{Myr}$ for UCL. Contrary to previous findings, it appears that LCC is coeval with, or slightly older than, UCL. The secular parallaxes of the Sco-Cen pre-MS stars yield distances of $85-215 \mathrm{pc}$, with 12 of the LCC members lying within $100 \mathrm{pc}$ of the Sun. Only 1 out of $110\left(0.9_{-0.8}^{+2.1 \%} ; 1 \sigma\right)$ pre-MS solar-type stars in the sample with ages of $13 \pm 1$ (s.e.) $\pm 6(1 \sigma)$ Myr and masses of $1.3 \pm 0.2(1 \sigma) M_{\odot}$ shows both enhanced $\mathrm{H} \alpha$ emission and a K-band excess indicative of accretion from a truncated circumstellar disk: the nearby $(d \simeq 86 \mathrm{pc}$ ) classical $\mathrm{T}$ Tauri star PDS 66.
\end{abstract}

Subject headings: Galaxy : open clusters and associations: individual (Sco OB2, Lower Centaurus-Crux, Upper Centaurus-Lupus) — stars: activity — stars: formation — stars: kinematics — stars: pre-main sequence - X-rays: stars

\section{Introduction}

Post-T Tauri stars (PTTSs) are low-mass, premain sequence (pre-MS) stars with properties intermediate between $\mathrm{T}$ Tauri stars found in molecular clouds (both "classical" with evidence for ac- cretion from a circumstellar disk and "weak-lined" lacking such evidence; CTTSs \& WTTSs; ages $<$ few Myr) and zero-age main sequence stars (ZAMS; ages > 30-100 Myr). Although strict observational criteria do not exist for classifying PTTSs as such, a working definition is a low- 
mass $\operatorname{star}\left(<2 M_{\odot}\right)$ that is Li-rich compared to stars in ZAMS open clusters such as the $\sim 120$ Myr-old Pleiades, and whose theoretical H-R position $\left(\log \mathrm{T}_{\text {eff }}\right.$ and $\left.\log \mathrm{L} / \mathrm{L}_{\odot}\right)$ is above the main sequence (Herbig 1978; Jensen 2001). Since these criteria also apply to CTTSs and WTTSs, one could argue that in addition, PTTSs should be located in regions devoid of nearby molecular gas or nebulosity. Classifying PTTSs by these criteria has complications: (1) few young field stars not associated with well-studied molecular clouds currently have accurately measured distances (hence known luminosities), (2) unresolved binarity can make stars with known distances appear more luminous, and thus younger, and (3) there is a dispersion in observed $\mathrm{Li}$ abundances among stars with the same masses and ages in coeval open clusters. Pre-main sequence stars exhibit considerable chromospheric $(\mathrm{H} \alpha, \mathrm{Ca} \mathrm{H} \& \mathrm{~K})$ and coronal Xray emission. Only a few PTTS candidates were known before the Einstein and ROSAT X-ray missions, and X-ray surveys have become the primary means of identifying these pre-MS stars.

Investigations of pre-main sequence evolution have been hampered by a lack of large samples of well-characterized PTTSs. This deficit has impacted studies of pre-MS angular momentum evolution (e.g. Rebull et al. 2001; Bouvier et al. 1997), stellar multiplicity (e.g. Köhler et al. 2000), and circumstellar disk evolution (e.g. Spangler et al. 2001; Haisch, Lada, \& Lada 2001). The nearest post- $\mathrm{T}$ Tauri stars also provide optimal targets for young exoplanet and brown dwarf searches (e.g. Lowrance et al. 2000). These objects are much more luminous early in their evolution, and the closest targets enable characterization of the smallest orbital radii. With a post$\mathrm{T}$ Tauri population in a nearby $\mathrm{OB}$ association, we can address basic questions such as: How long does star-formation persist in a giant molecular cloud? What is the duration of the accretion phase for young solar-type stars?

Identifying a bona fide PTTS sample can be accomplished by searching for low-mass members of nearby fossil OB associations. The Sco-Cen OB complex (Sco OB2) is the nearest OB association to the Sun (mean subgroup distances range from 118-145 pc; de Zeeuw et al. (1999), hereafter dZ99), and covers roughly 2000 square degrees $(\sim 5 \%)$ of the sky. The complex is comprised of three kinematic subgroups (Blaauw 1946) with nuclear ages ranging from 5 to $15 \mathrm{Myr}$, a molecular cloud currently undergoing star-formation (the $\rho$ Oph complex, Wilking, Lada, \& Young 1989; Blaauw 1991; de Geus 1992), and perhaps several smaller cloud complexes in the vicinity (e.g. the Lupus, Corona Australis, Chamaeleon, Musca, and Coalsack clouds). The three subgroups are Upper Scorpius (US; age 5-6 Myr), Upper Centaurus-Lupus (UCL; age 14-15 Myr), and Lower Centaurus-Crux (LCC; age 11-12 Myr de Geus et al. 1989). US has been studied extensively in recent years (e.g. Preibisch \& Zinnecker 1999, and references therein), however UCL and LCC have received relatively little attention.

In this work, we investigate the low-mass $\left(<2 M_{\odot}\right)$ membership of the two oldest Sco-Cen OB subgroups (LCC and UCL) utilizing recently available astrometric catalogs (Hipparcos, Astrographic Catalog-Tycho (ACT), Tycho Reference Catalog (TRC), and Tycho-2), the 2 Micron All Sky Survey (2MASS), and the ROSAT All-Sky Survey (RASS). We conduct a spectroscopic survey of two samples: (1) an X-ray-selected sample of late-type stars from the kinematic candidate membership lists of Hoogerwerf (2000), and (2) the G-K type Hipparcos members of the OB subgroups from dZ99. In $\S 2$, we discuss the procedure for selecting candidate pre-MS stars from both samples, and $\S 3$ discusses the observations and assembled database. $\S 4$ describes the data analysis and characterization of our stellar sample, and $\S 5$ discusses the selection of pre-MS stars, sample contamination, and completeness. $\S 6$ describes how we construct an H-R diagram for the subgroups, and $\S 7$ presents results regarding the ages of the subgroups, their age spreads, and the frequency of accretion disks around pre-MS stars. $\S 8$ discusses the star-formation history of LCC and UCL, and $\S 9$ summarizes the findings of our survey.

\section{Selection of Candidate Pre-MS Stars}

\subsection{The Hipparcos Sample}

DZ99 lists Hipparcos Sco-Cen members that were selected using both de Bruijne's (1999a) refurbished convergent point method and Hoogerwerf \& Aguilar's spaghetti method (1999). Their membership lists contained $31 \mathrm{G}-\mathrm{K}$ stars in UCL 
and 21 G-K stars in LCC (their Table C1). Most of these bright stars have been classified in the Michigan Spectral Survey (e.g. Houk \& Cowley 1975), however SIMBAD ${ }^{1}$ reveals that most have been studied no further. We limit the survey to the 30 G-K candidates with Michigan luminosity classes IV or V (see Table 1). Stars with borderline $\mathrm{F} / \mathrm{G}$ Michigan types were not observed. HIP 63962 and 73777 met the criteria, but were not observed. DZ99 estimated the contamination by G-K-type interlopers of all luminosity classes to be $32 \%$ for LCC and $24 \%$ for UCL. Of these 31 stars, 17 also have RASS-BSC X-ray counterparts within $40^{\prime \prime}$.

\subsection{The RASS-ACT/TRC Sample}

To identify lower-mass members of an OB association, one can search for stars whose proper motions are similar to those of high-mass members. The high-mass membership and moving group solution for each OB subgroup were determined by dZ99 and de Bruijne (1999b). Thousands of faint stars in the ACT and TRC astrometric catalogs ${ }^{2}$ were identified by Hoogerwerf (2000) as candidate low-mass LCC and UCL members. A high degree of contamination from interlopers is expected due to the similarity of the space motions of the subgroups to that of the Local Standard of Rest, compounded by the low galactic latitude of the subgroups. The selection of ACT/TRC candidate members is described in detail in $\S 4$ of Hoogerwerf (2000).

The Hoogerwerf ACT/TRC membership lists for LCC and UCL were slightly modified, and filtered, to produce the final target list. First, we requested from R. Hoogerwerf (personal communication) candidate membership lists with different color-magnitude constraints from that described in Hoogerwerf (2000). The new colormagnitude selection box is essentially a polygon defined by the Schmidt-Kaler (1982) empirical zero-age main sequence $\left(B-V\right.$ vs. $\left.\mathrm{M}_{V}\right)$ at the

\footnotetext{
${ }^{1}$ http://simbad.u-strasbg.fr/Simbad

${ }^{2}$ The ACT (Urban et al. 1998) and TRC catalogs (Høg et al. 1998) were used for target selection for this project in 1999, however we use the photometry and astrometry from the Tycho-2 catalog (available in 2000; Høg et al. $2000 \mathrm{a}, \mathrm{b})$ in the data analysis. The Tycho- 2 catalog was a joint USNO/Copenhagen project, and its data supercede the contents of the ACT and TRC catalogs.
}

mean distance for each subgroup (dZ99), where we take all stars $\Delta \mathrm{M}_{V}=3 \mathrm{mag}$ above and $\Delta \mathrm{M}_{V}=1$ mag below the ZAMS line. Hoogerwerf originally selected only those stars within $\Delta \mathrm{M}_{V}=1.5 \mathrm{mag}$ above the ZAMS, however this could inadvertently omit younger members or binaries. The selection box contained 1353 ACT and TRC stars in LCC, and 1874 stars in UCL. In order to target low-mass solar-type stars with G-K spectral types, we retained only those stars with Johnson $B-V$ $\geq 0.58 \mathrm{mag}$ (the unreddened color of G0 dwarfs; Drilling \& Landolt 2000). No red $B-V$ limit was imposed. After the color-magnitude selection, we retained only those stars which were identified as kinematic members in both the ACT and TRC astrometric catalogs. This final color-selection of the ACT/TRC lists resulted in 785 UCL candidates and 679 LCC candidates.

In order to further filter the target list, we selected only those ACT/TRC candidates which had ROSAT All-Sky Survey Bright Source Cata$\log$ (RASS-BSC) X-ray counterparts. Voges et al. (1999) cross-referenced the RASS-BSC with the Tycho catalog and found that $68 \%$ of the opticalX-ray correlations were within $13^{\prime \prime}$, and $90 \%$ of the correlations were within $25^{\prime \prime}$. In plotting a histogram of the separation distance between RASSBSC X-ray sources and ACT/TRC stars, we independently find $40^{\prime \prime}$ to be an optimal search radius. No constraints on X-ray hardness ratio were imposed in the target selection. In order to calculate X-ray luminosities, we assume the X-ray energy conversion factor for the ROSAT PSPC detector from Fleming et al. (1995). The linearity of this X-ray efficiency relation spans the temperature range of stellar coronae from inactive subdwarf stars to extremely active RS CVns and T Tauri stars. Unsurprisingly, the kinematic selection of ACT/TRC stars also selected many of the same stars as in the Hipparcos sample (HIP 57524, 59854, 62445, 65423, 66001, 66941, 67522, 75924, $76472,77135,77524,77656,80636)$. These stars are retained in the Hipparcos sample (Table 1), and omitted from the RASS-ACT/TRC list (Table 2). The final target list of 96 RASS-ACT/TRC stars (40 LCC, 56 UCL) is given in Table 2. 


\section{Observations}

Blue and red optical spectra of the pre-MS candidates were taken simultaneously with the DualBeam Spectrograph (DBS) on the Siding Springs 2.3-m telescope on the nights of 20-24 April 2000. The DBS instrument is detailed in Rodgers, Conroy, \& Bloxham (1988). Using a $2^{\prime \prime}$ wide slit, we used the $\mathrm{B} 600 \mathrm{l} / \mathrm{mm}$ grating in first order on the blue channel, yielding $2.8 \AA$ FWHM resolution from $3838-5423 \AA$. The red channel observations were done with the $\mathrm{R} 1200 \mathrm{l} / \mathrm{mm}$ grating in

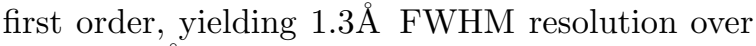

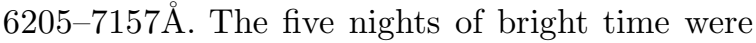
predominantly clear to partly cloudy. Signal-tonoise ratios of $\sim 50-200$ per resolution element were typically reached with integration times of 120 720 s. Flat-fields and bias-frames were observed at the beginning and end of each night. $\operatorname{NeAr} \lambda$ calibration arcs and spectrophotometric standards were observed every few hours. The spectra were reduced using standard IRAF routines. In order to remove low-order chromatic effects from the band-ratio measurements, we spectrophotometrically calibrated all of the target spectra using 2 standard stars from Hamuy et al. (1994). A total of 118 program stars (§2.1 and $\S 2.2)$ and $20 \mathrm{MK}$ spectral standards $(\S 4.1 .1)$ were observed. The major stellar absorption features of one of the single standard G stars were shifted to a zero-velocity wavelength scale. The spectra of all of the stars were then cross-correlated against this standard star using the IRAF task fxcor, and then shifted to the common, rest-frame wavelength scale. This was done to ensure proper identification of weak lines, as well as to make sure that the band-ratio measurements were sampling the same spectral range in each stellar spectrum.

\section{Analysis of Spectra}

\subsection{Spectral Types and Luminosity Clas- sification}

\subsubsection{Standard Stars}

We observed 20 spectral standards including dwarfs and subgiants (luminosity classes IV and V) and a few giants (III). A summary of their properties is listed in Table 3 . To permit quantitative examination of trends in the strengths of spectral features, as well as interpolation between spectral types, we adopt the numerical subtype scaling of Keenan (1984; i.e. here listed as "SpT", where $\mathrm{G} 0=30, \mathrm{G} 2=31, \mathrm{~K} 0=34$, etc.). All of the standard stars are classified on the MK system by Keenan \& McNeil (1989), except for HR 7061 (Garcia 1989). Table 3 also lists their spectral types as given in the Michigan Spectral Survey atlases of N. Houk (e.g. 1978). The sample standard deviation of a linear fit between the Keenan and Houk spectral types for dwarfs and subgiants, on Keenan's subtype scale, is $\sigma(\mathrm{SpT})=0.6$ subtypes. The $\sim 0.6$ subtype uncertainty probably represents the best that can be done using visual spectral types determined by different authors.

The adopted spectral types are those of Keenan \& McNeil's, however the luminosity classification was verified (and some times changed) by virtue of (1) position of the stars on a color-magnitude diagram based on Hipparcos data, (2) position in a temperature vs. Sr II $\lambda 4077 / \mathrm{Fe}$ I $\lambda 4071$ ratio diagram (see $\S 4.1 .3$, Fig. 1), and (3) published $\log g$ estimates. Although changing the classification of some standards may appear imprudent, the $\mathrm{H}-\mathrm{R}$ diagram positions, $\mathrm{Sr} / \mathrm{Fe}$ line ratio, and derived $\log g$ values (Cayrel de Strobel et al. 2001) are all consistent with our new adopted luminosity classes $^{3}$. In every case the difference was only half of a luminosity class, and only $5 / 20$ of the stars were changed. Notes on the revised luminosity classifications are given in Appendix B.

\subsubsection{Visual Classification}

The blue spectrum of each star was assigned a spectral type visually by E.M. through comparison with the standards in Table 3 . In order to distinguish subtypes, we focused on several features such as the $\mathrm{G}$ band $(\lambda 4310)$, Ca I $\lambda 4227, \mathrm{Cr}$ I $\lambda 4254$ and nearby Fe lines, and the $\mathrm{Mg}$ b lines $\lambda 5167, \lambda 5173$, $\lambda 5184$. Balmer lines were ignored due to possible chromospheric emission. After making an initial guess through comparison with a wide range of spectral types, a final visual spectral type was assigned through comparison to standards within \pm 2 subtypes of the initial guess.

To test the accuracy of our visual classification, we compared our spectral types to those of qual-

\footnotetext{
${ }^{3}$ Hipparcos data also led Keenan \& Barnbaum (1999) to revise the luminosity classes of a few giant star standards at the half luminosity class level.
} 
ity 1 or 2 in the Michigan Spectral Survey. The average difference is not significant: $-0.4 \pm 0.6(1 \sigma$ sample standard deviation) subtypes (on Keenan's scale). The 12 Hipparcos stars were later visually typed a second time. Between the two estimates for each star, we estimate that the $1 \sigma$ uncertainty in our visual spectral types is 0.6 subtypes. This is comparable to the dispersion between the Keenan and Houk spectral types for the standards themselves.

\subsubsection{Quantitative Spectral Type Estimation}

A two-dimensional quantitative spectral type (subtype plus luminosity class) can be estimated using integrated fluxes over narrow bands sensitive to temperature and surface gravity. We tested various ratios defined by Malyuto \& Schmidt-Kaler (1997) and Rose (1984) for this purpose, as well as from Gray's spectral atlas (2000). In testing bandratios as temperature indicators for our standards, we noticed that some had slight surface gravity dependencies. A surface gravity dependence in our temperature indicators could systematically affect our $T_{\text {eff }}$ estimates. We first discuss our surface gravity indicator and then define our temperature estimators using only subgiant and dwarf standards, thus mitigating the effects of surface gravity.

The most widely used surface gravity diagnostic for $\mathrm{G}$ and $\mathrm{K}$ stars is the ratio between Sr II $\lambda 4077$ and nearby Fe lines (e.g. Keenan \& McNeil 1976; Gray 2000). In thin and thick disk dwarfs, the abundance ratio $[\mathrm{Sr} / \mathrm{Fe}]$ is within $\sim 0.1$ dex of solar for most stars (Mashonkina \& Gehren 2001). A quantitative surface gravity (luminosity class) indicator was established by Rose (1984) from lowresolution spectra using the maximum absorption line depth for Sr II $\lambda 4077$ and the average for the atomic Fe $\lambda 4045$ and $\lambda 4063$ lines. We measure the fluxes in $3 \AA$ bands centered on the Sr II $\lambda 4077$ line and the Fe I $\lambda 4071$ line. Ratios between the $\lambda 4077$ line and the other nearby Fe lines $(\lambda 4045$, $\lambda 4063)$ did not distinguish subgiants and giants.

For a temperature estimator, we adopted Index 6 of Malyuto \& Schmidt-Kaler (1997) ( $\lambda \lambda 5125$ $5245 / \lambda \lambda 5245-5290)$ hereafter referred to as "MI6". The temperature sensitivity of this indicator largely reflects differing amounts of line-blanketing in these two wavelength regimes - mainly by the $\mathrm{Mg}$ b lines $(\lambda 5167, \lambda 5173$, and $\lambda 5184)$, and many
Fe lines (e.g. Fe I 15270). Although the $\mathrm{Mg}$ b lines are somewhat surface gravity sensitive, within the $\log g$ and $T_{\text {eff }}$ regime of our standards and program stars, the temperature sensitivity is dominant. The difference in central wavelength between the two bandpasses is only $82 \AA$, and the effects of reddening are negligible (Mathis 1990). For a temperature indicator, we fit a low-order polynomial to MI6 vs. SpT for the dwarf and subgiant standard stars (see Appendix C) which has a $1 \sigma$ sample standard deviation of 0.6 subtypes.

Fig. 1 plots the temperature-sensitive MI6 index versus our surface gravity discriminant (Sr II $\lambda 4077 / \mathrm{Fe}$ I $\lambda 4071)$. The dwarf standards form a very narrow sequence in Fig. 1, confirming the lack of cosmic scatter in $[\mathrm{Sr} / \mathrm{Fe}]$ values among field stars and the insensitivity of logg to spectral type for $G-K$ dwarfs. The polynomial fit to the dwarf data is given in Appendix C. There is a gap between the dwarf and subgiant loci between $\sim 1-2.5 \sigma$ (sample standard deviation) of the dwarf locus polynomial, and we set the subgiant/dwarf separation at $2 \sigma$. We classify stars within $2 \sigma$ of the solid dwarf line in Fig. 1 as dwarfs (4 of 96 RASS-ACT/TRC stars, 4 of 20 HIP stars), and three stars near the giant locus (TYC 8992-605-1, HIP 68726, and HIP 74501) as giants. We classify the rest as subgiants.

Gray (2000) suggests Y II $\lambda 4376 /$ Fe I $\lambda 4383$ as a surface gravity indicator for late-G stars using low-resolution spectra. From the solar spectral atlas of Wallace, Hinkle, \& Livingston (1998), it appears that Gray's low-resolution Y II $\lambda 4376$ feature is actually a blend of several lines of nearly equal strength. In order to test the properties of this band-ratio, we measure the flux in $3 \AA$ windows centered on wavelengths $4383.6 \AA$ and

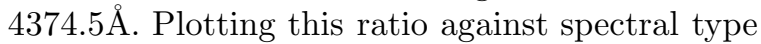
for the standard stars showed a very tight locus for the dwarfs, however luminosity classes IV-V, IV, and III were indistinguishable from the dwarfs and each other. We found this ratio unsuitable for the purposes of luminosity classification of our targets, but we find it to be an excellent temperature estimator for FGK dwarfs, subgiants, and giants. Among the 20 standards, the measurement of the $\lambda 4374 / \lambda 4383$ band-ratio vs. spectral type gives a tight correlation (sample standard deviation $1 \sigma=$ 0.6 subtypes). We adopt the $\lambda 4374 / \lambda 4383$ band ratio as our third, independent estimator of spec- 
tral type (polynomial fit is given in Appendix C).

\subsubsection{Final Spectral Types}

The three temperature-type estimates agree well for the majority of the program stars. The mean difference between the MI6 and visual spectral types is 0.7 subtypes. The mean difference between the $\lambda 4374 / \lambda 4383$ band-ratio types and the visual types is 0.6 subtypes. We calculate a mean spectral type and standard error of the mean using the three classifications. The mean is unweighted since all three relations appeared to have $1 \sigma$ sample standard deviations of $\approx 0.6$ subtypes in their accuracy. The average standard error of the mean is 0.5 subtypes. We believe that using multiple techniques mitigates the effects that rapid rotation, binarity, etc. can introduce into visual classification alone. The spectral types are listed in Tables 6,5 and 7.

\subsection{Additional Spectroscopic Diagnostics}

\subsubsection{Chromospheric Ha Emission}

Medium-to-low resolution spectra of chromospherically active stars show the $\mathrm{H} \alpha$ line to be partially filled-in, or even fully in emission. We measure the EW of the entire $\mathrm{H} \alpha$ feature; our resolution is insufficient to separate the "core" chromospheric emission from the photospheric $\mathrm{H} \alpha$ absorption line. A significant number of our stars show $\mathrm{H} \alpha$ emission (19\% of the RASS-ACT/TRC G-K type stars). We characterize our targets stars as chromospherically active or inactive through comparing the $\mathrm{H} \alpha \mathrm{EW}$ to that of standards of identical spectral type. Fig. 2 shows the $\mathrm{EW}(\mathrm{H} \alpha)$ data for our targets and standard stars. Stars more than $2 \sigma$ above the dwarf/subgiant $\mathrm{EW}(\mathrm{H} \alpha)$ relation (a quadratic regression; see Appendix C) are considered to be active. The stars with $\mathrm{H} \alpha$ in emission (negative EWs) have an "e" appended to their spectral types in Tables 6,5 and 7 . The $\mathrm{H} \alpha$ EWs for each star are also listed in these tables.

\subsubsection{Li I $\lambda 6707$ Equivalent Width}

The presense of strong $\mathrm{Li}$ absorption in the spectra of late-type stars is a well-known diagnostic of stellar youth. Because of the extended timescale for significant $\mathrm{Li}$ depletion in stars of $\sim 1 \mathrm{M}_{\odot}$, strong Li absorption is necessary, but not sufficient indicator of pre-MS nature for $G$ stars.
However it is a powerful age descriminent when combined with our surface gravity indicator.

Many studies have shown that the equivalent width (EW) of Li I $\lambda 6707$ can be overestimated at low spectral resolution (e.g. Covino et al. 1997), especially for G-K stars. With a resolution of $1.3 \AA$, we consider our EWs to be approximate. The EWs were measured with Voigt profiles in the IRAF routine splot. The continuum level was estimated from nearby pseudo-continuum peaks. We subtract the contribution from the neighboring Fe I $\lambda 6707.4 \AA$ feature using the prescription of Soderblom et al. (1993). In order to test the validity of our Li EWs, we divided several of our Li-rich targets by standard stars of the same spectral type. The ratioed spectra exhibit only a major absorption feature at $\lambda 6707$. The division also removes the effects of blending by Fe lines (assuming the same stars have similar EWs). The EWs of this feature in the divided spectra corresponded well with our previous measurements, however the uncertainties in the EW appear to be $\sim 20-50 \mathrm{~m} \AA$ (with the maximum value being for spectroscopic binaries).

Fig. 3 shows the Li I $\lambda 6707$ EWs for our RASS$\mathrm{ACT} / \mathrm{TRC}$ and Hipparcos targets, separated according to their luminosity class (§4.1.3). Effective temperatures $\left(T_{\text {eff }}\right)$ come from the final spectral type (see $\S 6.2$ ). Most points lie above the Li I $\lambda 6707$ EWs that characterize young open clusters, plotted as low-order polynomial fits for the IC 2602 (30 Myr; Randich et al. 1997), Pleiades (70125 Myr; Soderblom et al. 1993; Basri, Marcy, \& Graham 1996), and M34 clusters (250 Myr; Jones et al. 1997). The comparison is not completely fair, however, since the cluster ZAMS stars will be roughly $10 \%$ less massive than the corresponding pre-MS stars. Even if most of our program stars were older ZAMS stars, they still would be Li-rich compared to stars in the well-studied open clusters. We select as "Li-rich" those stars above the solid line in Fig. 3. Considering the uncertainties in our $\mathrm{EW}(\mathrm{Li})$ measurements, and the lack of any other $\sim 10-20$ Myr-old pre-MS G-K-type stellar samples with which to compare, we are not compelled to subdivide our sample further. We will reserve a more detailed investigation of the $\mathrm{Li}$ abundances for a future high-resolution spectral study. For the present, we are content to have demonstrated that we have identified a popula- 
tion which appears to be more Li-rich than ZAMS stars.

\section{Defining the PTTS Sample}

\subsection{Membership Status}

Our survey was designed to identify the preMS G and K-type stars in the Sco-Cen OB association. We classified the late-type stars according to their positions in Figs. 1, 2 and 3 (Table 4). We consider the 110 stars (85/96 RASS-ACT/TRC and 16/30 Hipparcos) classified as "Li-rich", "subgiant", and "active" as bona fide post-T Tauri stars ("pre-MS"). Li-rich stars with subgiant surface gravities and $\mathrm{H} \alpha \mathrm{EWs}$ similar to the standard field stars (i.e. "inactive") are called "pre-MS?". Only 3 of the RASS-ACT/TRC stars, and 6 of the HIP stars are classified as "pre-MS?". The lone object with giant-like surface gravity in the RASSACT/TRC X-ray-selected sample (TYC 8992-6051 ) is Li-rich, and we also classify it as a pre-MS PTTS. The 9 "pre-MS?" stars were included in our statistics concerning the star-formation history and disk-frequency of the sample $(\S 7)$ for a total of 110 candidate lower-mass members of the LCC and UCL subgroups. All 13 stars selected in the RASS-ACT/TRC sample which overlaped with dZ99's membership lists were found to be pre-MS candidates. Our RASS-ACT/TRC sample (including the 13 dZ99 stars also selected) yielded a pre-MS hit-rate of $(88+13) /(96+13)=$ $93 \%$. Of the 30 dZ99 candidates we observed, $22 / 30(73 \%)$ are classified as pre-MS or "preMS?". The numbers of stars by membership class are listed in Table 4. Pre-MS stars in the Hipparcos sample are listed in Table 5, and those in the RASS-ACT/TRC sample are given in Table 6. Li-rich stars with dwarf-like surface gravity ( $\mathrm{N}$ $=5$ ) were considered young main sequence field stars ("ZAMS"), and are listed along with other interlopers in Table 7.

\subsection{Sample Contamination}

The primary contaminants one would expect from an X-ray- and proper motion-selected sample are X-ray-luminous ZAMS stars (ages $\approx 0.1-1$ Gyr). Field ZAMS stars could occupy the same region of UVW velocity space, and be selected in our study by virtue of their proper motions and Xray emission. However, our selection of candidate
Sco-Cen members utilizes a surface gravity criterion which should minimize contamination. Even if our surface gravity indicator was in error, we claim ZAMS stars do not dominate our sample. Field ZAMS stars exhibit a large spread in Li EWs (especially for the late-G and early-K stars), however this is not observed in Fig. 3. The star just below the "Li-rich" line in Fig. 3 (TYC 7318-5931; $\mathrm{G} 9, \mathrm{EW}(\mathrm{Li}) \simeq 150 \mathrm{~m} \AA$ ) happens to be the sole RASS-ACT/TRC star with inferred $\log g$ higher than that of the dwarf standards in Fig. 1. We consider TYC 7318-593-1 to be a field ZAMS star candidate due to its intermediate Li strength and high surface gravity.

We can rule out most of the pre-MS candidates being Li-rich post-MS stars. Based on the surveys of $\mathrm{Li}$ abundances in field subgiants by Randich et al. (1999) and Pallavicini et al. (1987), we do not expect to find any post-MS subgiants with $\operatorname{EW}(\lambda 6707)>100 \mathrm{~m} \AA$. Even if our measured EWs for the Li I $\lambda 6707$ line are over-estimated due to low spectral resolution, the overestimate would have to be greater than a factor of two to reconcile our sources with even the most Li-rich subgiants found in the Randich et al. survey. Our spectral analysis suggests that the majority of our sample stars are both Li-rich and above the main sequence (i.e. pre-MS).

Could some of our stars be post-MS chromospherically active binaries (CABs) or RS CVn systems? The light from an RS CVn system would be dominated by a rapidly rotating, evolved (subgiant) primary. Only six of our targets are Lipoor subgiants (HIP 63797, 81775, TYC 829392-1, 7833-1106-1, 7858-526-1, and 8285-847-1). The first three appear to be normal subgiants. TYC 7833-1106-1 is possibly a spectroscopic binary. TYC 7858-526-1 has a wide, broad $\mathrm{H} \alpha$ absorption line. It appears to be a multiple late-F star (we classify it as F8.5; Houk (1982) classify it as F5), so the star could hide a cosmic Li abundance due to the increased ionization of $\mathrm{Li} \mathrm{I}$ in $\mathrm{F}$ stars (and correspondingly lower $\mathrm{EW}(\mathrm{Li})$ ). The system could be a legitimate member, but we exclude it from the pre-MS sample. The subgiant TYC 8285-847-1 (HIP $69781=$ V636 Cen) is probably a CAB. It is a previously known grazing, eclipsing binary (e.g. Popper 1966) and its saturated X-ray emission argues for being a true CAB. Finally, the Li-rich star TYC 8992-605-1 
(star \#19; K0+III) is the only RASS-ACT/TRC star that appears in the giant regime of Fig. 1. The star is an obvious spectroscopic binary of nearly equal mass. We believe this star is probably a pre-MS binary, and include it in our "preMS?" sample. It appears that CABs are a negligible contaminant when using X-ray and kinematic selection in tandem with medium dispersion spectroscopy to identify pre-MS populations.

\subsection{Sample Completeness}

We can make a rough estimate how many stars our selection procedure should have detected by counting the number of massive Sco-Cen members in a certain mass range, and assuming an initial mass function. We assume a complete membership census within a limited mass range (the revised B-star Hipparcos membership from dZ99), and then extrapolate how many stars we should have seen in our survey. We produce a theoretical H-R diagram for the subgroups' B stars (discussed at length in $\$ 7.2$ ), and calculate masses from the evolutionary tracks of Bertelli et al. (1994) $(\mathrm{Z}=0.02)$. We choose $2.5 M_{\odot}$ as our lower mass boundary (roughly the lower limit for B stars), and adopt $13 M_{\odot}$ as the upper mass boundary (slightly higher than the highest inferred mass from the main sequence members). In this mass range, we count $32 \mathrm{LCC}$ members and $56 \mathrm{UCL}$ members. We use a Kroupa (2001) IMF to predict how many low-mass stars might belong to the $\mathrm{OB}$ subgroups. Down to the hydrogen-burning limit $\left(0.08 M_{\odot}\right)$, a total population of $1200_{-300}^{+200}$ stars in LCC and $2200 \pm 300$ stars in UCL is predicted (Poisson errors) ${ }^{4}$. Between 1.1-1.4 $M_{\odot}$, the mass range of a 15 Myr-old population that our survey can probe (see $\S 7.1$ and Fig. 6), the Kroupa IMF predicts a population of $29_{-5}^{+6}$ stars in LCC, and $51_{-7}^{+8}$ stars in UCL. In this mass range, our survey detects 36 pre-MS stars in LCC and 40 preMS stars in UCL. The number of observed preMS stars with 1.1-1.4 $M_{\odot}$ corresponds to $+1.1 \sigma$ and $-1.6 \sigma$ of the predicted number, for LCC and UCL respectively. This suggests that our survey is fairly complete for LCC, but we might be missing $\sim 10$ members with masses of 1.1-1.4 $M_{\odot}$ in the more distant UCL subgroup if the subgroup mass

\footnotetext{
${ }^{4}$ For low-number statistical uncertainties, we use the $1 \sigma$ values from Gehrels (1986) throughout.
}

function is consistent with the field star IMF. The missing members of the UCL OB subgroup could be X-ray faint $\left(\mathrm{L}_{X} \leq 10^{30.2} \mathrm{erg} \mathrm{s}^{-1}\right)$ stars which we were capable of detecting in the closer LCC subgroup. The IMF extrapolation does suggest that we have likely found at least the majority of stars in this mass range in both OB subgroups (if not a complete census for LCC) and that our samples are representative of the total population.

\section{The H-R Diagram}

In order to investigate the star-formation history of the LCC and UCL OB subgroups, we convert our observational data (spectral types, photometry, distances) into estimates of temperature and luminosity. We then use theoretical evolutionary tracks to infer ages and masses for our stars.

\subsection{Photometry}

The primary sources of photometry for our sample of association member candidates are the Tycho-2 catalog (Høg et al. 2000a,b) and 2MASS working database. However, the Tycho and 2MASS bandpasses are non-standard, and must be converted to standard photometric systems to enable comparison with intrinsic colors of normal stars and the interstellar reddening vector. To convert the Tycho photometry to the Johnson system, we fit low-order polynomials to the data in Table 2 of Bessell (2000) (relations given in Appendix $\mathrm{C}$ ). A caveat is that Bessell's calibrations are for $\mathrm{B}-\mathrm{G}$ dwarfs and $\mathrm{K}-\mathrm{M}$ giants. The majority of our stars appear to be pre-MS G-K stars, whose intrinsic colors should more closely match those of dwarfs rather than giants. To convert the 2MASS $J H K_{s}$ data to the system of Bessell \& Brett (1988), we use the conversions of Carpenter (2001). The original optical and near-IR photometry for our target stars is given in Tables 2 and 1.

\subsection{Temperature Scale}

To fix stellar properties as a function of spectral type, we adopt relations (i.e. intrinsic colors, BCs) from Table A5 of Kenyon \& Hartmann (1995). Previous studies have shown that colors and BCs as a function of $T_{\text {eff }}$ are largely independent of surface gravity over the range of interest for this study (e.g. Bessell, Castelli, \& Plez 
1998). However, $T_{\text {eff }}$ decreases with lower $\log g$ for FGK stars. After some investigation (see Appendix A), we decided to adopt the dwarf $T_{\text {eff }}$ scale of Schmidt-Kaler (1982) (which Kenyon \& Hartmann (1995) also use) with a -35 K offset to account for the effects of lower $\log g$ in our sample stars. The scatter in published dwarf $T_{\text {eff }}$ scales is $60 \mathrm{~K}(1 \sigma)$ among $\mathrm{G}$ stars, so while the shift is systematic, its magnitude is of the order of the uncertainties. The uncertainties in $T_{\text {eff }}$ given in column 9 of Tables 5 and 6 include the uncertainty in spectral type and the scatter in published $T_{\text {eff }}$ scales. The typical $1 \sigma$ uncertainties in $T_{\text {eff }}$ for the pre-MS stars is $\approx 100 \mathrm{~K}$.

\subsection{Secular Parallaxes}

All of the stars in our sample have published proper motions, but only a few dozen have trigonometric parallaxes measured by Hipparcos. The stars are distributed over hundreds of square degrees of sky, and inhabit stellar associations which are tens of parsecs in depth. Adopting a standard distance for all of the stars in the association introduces unwanted scatter in the H-R diagram. With accurate proper motions available, we calculate individual distances to the pre-MS candidates using moving cluster or "secular" parallaxes (e.g. Smart 1968). We adopt the equations and formalism of de Bruijne (1999b), as well as his space motions and convergent points for the LCC and UCL OB subgroups. The uncertainties in the secular parallaxes are dominated by the uncertainties in the proper motion $\left(\sigma_{\pi} \propto \sigma_{\mu}\right)$, but contain a term added in quadrature accounting for a projected $1 \mathrm{~km} \mathrm{~s}^{-1}$ internal velocity dispersion (see $\S 4$ of de Bruijne 1999b). The secular parallax is only meaningful if the star is indeed a member of the group. Our spectroscopic survey has confirmed that most of the candidate stars are legitimately pre-MS, and that they are most likely members of the OB subgroups. Secular parallaxes for older, interloper stars are meaningless and ignored. In Tables 5 and 6 , we list the secular parallaxes and membership probabilities for the preMS stars in our survey. We calculate membership probabilities $\mathrm{P}_{1}$ and $\mathrm{P}_{3}$ (using formulae 4 and 6 from dZ99), which have assumed internal velocity dispersions of $1 \mathrm{kms}^{-1}$ (de Bruijne 1999b) and $3 \mathrm{~km} \mathrm{~s}^{-1}$ (dZ99), respectively.

The robustness of our method can be illustrated
(Fig. 4) by comparing the secular parallaxes $\left(\pi_{s e c}\right)$ to the Hipparcos trigonometric parallaxes $\left(\pi_{H I P}\right)$. The uncertainties are typically 1-2 mas for the Hipparcos parallaxes, and 0.5-1 mas for our secular parallax estimates. The secular and trigonometric parallaxes agree quite well for the few pre-MS stars in our sample for which Hipparcos measured the parallax. The secular parallaxes yield distance uncertainties of $\sim 5-15 \%$ for most of the pre-MS stars.

\subsection{Luminosities}

With five-band photometry, a temperature / spectral type estimate, and a secular parallax, we calculate stellar luminosities for the pre-MS candidates. We adopt the absolute bolometric magnitude of the Sun $\left(M_{\mathrm{bol} \odot}=4.64\right)$ from SchmidtKaler (1982). In order to compromise between the uncertainties in luminosity due to reddening, photometric uncertainties, and possible K-band excess, we calculate the $M_{\text {bol }}$ using the dereddened 2MASS J magnitude. We estimate the visual extinction from a weighted mean of $\mathrm{A}_{V}$ estimates from the color excess in $B-V$ and $V-J$. We took the $\mathrm{E}(B-V)$ formula from Drilling \& Landolt $(2000)$ and the value of $\mathrm{A}_{J} / \mathrm{A}_{V}(=0.294)$ was taken from the near-IR extinction law of Mathis (1990) for a central wavelength $1.22 \mu \mathrm{m}$. The reddening $\mathrm{A}_{J}$ typically ranged from 0 to $0.35 \mathrm{mag}$ with formal uncertainties of $\sim 0.1$ mag. The typical uncertainty in $\log \mathrm{L} / \mathrm{L}_{\odot}$ for the pre-MS candidates is $\approx 0.08$ dex. With the luminosities and $\mathrm{X}$-ray fluxes from the RASS BSC catalog (Voges et al. 1999), we calculate the ratio of $\mathrm{X}$ ray/bolometric radiation for the stars with X-ray counterparts. The derived values of $\log \left(\mathrm{L}_{X} / \mathrm{L}_{b o l}\right)$ are in the range of $10^{-2.8}-10^{-3.8}$, indicating coronal X-ray emission elevated above most ZAMS Gtype stars (e.g. Pleiads; Stauffer et al. 1994).

\subsection{Evolutionary Tracks}

In order to infer theoretical masses and ages from our pre-MS candidates, we use the evolutionary tracks from D'Antona \& Mazzitelli (1997) $\left(\mathrm{DM} 97 ; \mathrm{Z}=0.02, \mathrm{x}_{D}=2 \times 10^{-5}\right)$, Siess, Dufour, \& Forestini (2000) (SDF00; $Z=0.02$ ), and Palla \& Stahler (2001) (PS01). Ages and masses for a given $\log \mathrm{T}_{\text {eff }}$ and $\log \mathrm{L} / \mathrm{L}_{\odot}$ were calculated using an interpolation algorithm. Given the mean observational errors $\left(\sigma\left(\log T_{e f f}, \log \mathrm{L} / \mathrm{L}_{\odot}\right)\right.$ 
$=0.007,0.078$ dex for LCC Pre-MS stars, and $\sigma\left(\log T_{\text {eff }}, \log \mathrm{L} / \mathrm{L}_{\odot}\right)=0.009,0.084$ dex among UCL Pre-MS stars), we estimate the isochronal age uncertainties for an individual star to be approximately 4, 5, 7 Myr (DM97, PS01, SDF00) in LCC, and 4, 5, 5 Myr (DM97, PS01, SDF00) in UCL, as illustrated in Fig. 5. The uncertainties in the interpolated masses are $0.1 \mathrm{M}_{\odot}$ for all three sets of tracks. Fig. 6 shows the H-R diagram for the pre-MS candidates overlayed with the evolutionary tracks of DM97.

\section{Results}

The ages of the low-mass population of LCC and UCL have not been estimated before, though de Geus et al. (1989) and de Zeeuw \& Brand (1985) give main sequence turn-off ages. In $\S 7.1$ we estimate the pre-MS ages for the two subgroups, and put an upper limit on the intrinsic age spread. In $\S 7.2$ we calculate new turn-off ages for the subgroups using early B stars from the revised Hipparcos membership lists of dZ99.

\subsection{Pre-MS Ages and Age Spread}

The H-R diagram for our "pre-MS" and "preMS?" stars is shown in Fig. 6, overlayed with the evolutionary tracks of D'Antona \& Mazzitelli (1997). The temperatures and luminosities of the pre-MS stars are given in Columns 9 and 10 of Tables 6 and 5, along with their inferred masses and ages (columns 15 through 17). One notices immediately that the bulk of isochronal ages are in the range of $\sim 10-20 \mathrm{Myr}$. The age range is nearly identical for both groups. To assess the effects of our magnitude limit in biasing our mean age estimates, in Fig. 7 we plot the mean preMS age (with standard errors of the mean) for the pre-MS subgroup samples as a function of minimum $\log \mathrm{T}_{\text {eff }}$ cut-off. The magnitude bias of our survey is clearly apparent: the mean age systematically decreases when stars with $\log \mathrm{T}_{\text {eff }}<3.73$ are included in the calculation. In calculating the pre-MS ages of the OB subgroups, we explicitly omit the pre-MS stars with $\log \mathrm{T}_{\text {eff }}<3.73(30 \%$ of our sample). This temperature threshold intersects our magnitude limits at ages of $\sim 25 \mathrm{Myr}$ for stars of $1 M_{\odot}$ on the DM97 tracks. That the lines in Fig. 7 are nearly flat for $\log \mathrm{T}_{\text {eff }}>3.73$, suggests that (detectable) stars with ages of $>25 \mathrm{Myr}$ are not a significant component of either subgroup (also see discussion in §8.1).

Fig. 8 displays histograms of the isochronal ages for the pre-MS stars in the LCC and UCL subgroups derived using DM97 and SDF00 evolutionary tracks. These tracks represent the extrema in age estimates for our sample (DM97 is youngest, SDF00 is oldest). The $1 \sigma$ age dispersion among the unbiased samples $\left(\log \mathrm{T}_{\text {eff }}>3.73\right)$ is 5-9 Myr for both groups. If we remove the known spectroscopic binaries (see notes in Tables 6 and 5), the age dispersions are 4-8 Myr. Because there may be additional unresolved binaries, this observed age spread places an upper limit on the intrinsic age spread. As illustrated in Fig. 5, the individual H-R diagram positions of the pre-MS samples have $\log \mathrm{T}_{\text {eff }}$ and $\log \mathrm{L} / \mathrm{L}_{\odot}$ errors which fold onto the evolutionary tracks with age uncertainties of 4-7 Myr. From this analysis, we conclude that the intrinsic $1 \sigma$ age dispersions in each subgroup must be less than $2-8 \mathrm{Myr}$ (i.e. $\sim 2 / 3$ rds of the star-formation took place in $<4-16 \mathrm{Myr}$ ). Using the DM97 pre-MS ages, which agree best with the turn-off age estimates $(\S 7.2)$, we find intrinsic $1 \sigma$ age dispersions of $2 \mathrm{Myr}$ (LCC) and 3 Myr (UCL).This implies that $68 \%$ of the low-mass star-formation took place within <4-6 Myr, and 95\% within $<8$-12 Myr in the OB subgroups. Our observational uncertainties and lack of knowledge about the unseen binarity of the pre-MS sample stars do not allow us to constrain the age spread more precisely than this. The mean age estimates for our unbiased pre-MS samples $\left(\log T_{\text {eff }}>3.73\right.$, SBs removed) are shown in Table 8. Counter to previous studies, we find that LCC is slightly older than UCL by 1-2 Myr (at 1-3 $\sigma$ significance), independent of which evolutionary tracks we use. From Fig. 8, we also conclude that star-formation ceased approximately $\sim 5-10$ Myr ago in the subgroups.

\subsection{Turn-off Ages}

De Geus et al. (1989) published the most recent age estimates for the LCC and UCL groups, but in light of the new Hipparcos distances and subgroup membership lists, we feel it is worthwhile reevaluating the subgroups' turn-off ages. We construct a theoretical H-R diagram for the B-type subgroup members of UCL and LCC listed both in Table C1 of dZ99 and Tables A2 and A3 
of de Bruijne (1999b). Several of the "classical" ${ }^{5}$ members rejected as members by Hipparcos from dZ99 are included as well. For input data, we use the following databases in order of availability: (1) ubvy $\beta$ photometry from the database of Hauck \& Mermilliod (1997), (2) UBV photometry from Slawson, Hill, \& Landstreet (1992), and (3) $U B V$ photometry from SIMBAD. For distances we use the secular parallaxes $\left(\pi_{s e c}\right)$ given in column 4 of Tables A2 and A3 of de Bruijne (1999b) when available, or the Hipparcos parallaxes $\left(\pi_{H I P}\right)$. We deredden the stars with ubvy $\beta$ photometry to the B-star sequence of Crawford (1978) using the prescription of Shobbrook (1983). For stars with Stromgren photometry, we calculate $T_{\text {eff }}$ using the temperature relation of Napiwotzki, Schönberner, \& Wenske (1993). If no $u b v y \beta$ photometry was available, we use $U B V$ photometry to calculate the reddening-free index $Q$ (Crawford \& Mandwewala 1976) to infer the star's unreddened color. A polynomial fit to Table 15.7 from Drilling \& Landolt (2000) is used to calculate $T_{\text {eff }}$ as a function of $(B-V)_{0}$. The $\mathrm{BC}$ versus $T_{\text {eff }}$ relation of Balona (1994) is used for all stars. We linearly interpolate between the isochrones from Bertelli et al. (1994) $(\mathrm{Y}=0.28$, $\mathrm{Z}=0.02$, convective overshoot) to infer ages for the subgroup B stars. The theoretical H-R diagram is shown in Fig. 9.

UCL has a well-defined MS turn-off composed of the Hipparcos members HIP 67464 ( $\nu$ Cen; B2IV), HIP 68245 ( $\phi$ Cen; B2IV), HIP 68282 $\left(\nu^{1}\right.$ Cen; B2IV-V), HIP 71860 ( $\alpha$ Lup; B1.5III), HIP 75141 ( $\delta$ Lup; B1.5IV), HIP 78384 ( $\eta$ Lup; B2.5IV), and HIP 82545 ( $\mu^{2}$ Sco; B2IV), as well as "classical" members (but Hipparcos non-members) HIP $82514\left(\mu_{1}\right.$ Sco; B1.5Vp) and 73273 ( $\beta$ Lup; B2III) ${ }^{6}$. The variable star HIP 67472 ( $\mu$ Cen; B2Vnpe) was excluded. Using the Bertelli et al. (1994) tracks, the mean age of the 7 turn-off Hipparcos members is $17 \pm 1 \mathrm{Myr}$. Including the two "classical" members has negligible effect on the mean age estimate. Our MS turn-off age estimate

\footnotetext{
5 "Classical" members are early-type stars which were included in Sco-Cen membership lists before the Hipparcos studies of dZ99.

${ }^{6}$ Note that the two Hipparcos non-members were found to be probable members by Hoogerwerf (2000) if the longbaseline ACT (HIP 73273) and TRC (HIP 82514) proper motions were used instead of the Hipparcos values.
}

for UCL is slightly older than de Geus et al.'s (14$15 \mathrm{Myr})$, and is close to the mean pre-MS ages that we found in $\S 7.1$ (15-22 Myr).

LCC lacks a well-defined turn-off, however we have enough early-B stars in the middle of their MS phase with which to make an age estimate. We estimate the age for LCC from the following main sequence B stars: HIP 59747 ( $\delta$ Cru; B2IV), HIP 60823 ( $\sigma$ Cen; B2V), HIP 61585 ( $\alpha$ Mus; B2IVV), HIP 63003 ( $\mu^{1} \mathrm{Cru}$; B2IV-V), and HIP 64004 $\left(\xi^{2}\right.$ Cen; B1.5V). The mean age for these five stars is $16 \pm 1 \mathrm{Myr}$; similar to what we found for UCL, and it agrees well with the younger end of our preMS age estimates (17-23 Myr). This age estimate is significantly older than previous estimates (10$12 \mathrm{Myr}$ ), and warrants more critical examination $(\S 8.3)$.

The new results yielded by our age analysis of the OB subgroups are: (1) two-thirds of the lowmass star-formation in each subgroup took place in less than a $\sim 5 \mathrm{Myr}$ span (and $95 \%$ took place within $\sim 10 \mathrm{Myr}$ ), (2) the pre-MS and B-star ages for LCC and UCL are in approximate agreement, (3) the B-star subgroup memberships defined by Hipparcos have ages of $16 \pm 1 \mathrm{Myr}$ and $17 \pm 1 \mathrm{Myr}$ for LCC and UCL, respectively. We discuss the implications of these results in $\S 8$.

\subsection{The Census of Accretion Disks}

An important question both for star and planet formation is the lifetime of accretion disks around young stars. Statistics for the frequency of active accretion disks around low-mass stars come predominantly from near-IR surveys of young associations and clusters (Hillenbrand \& Meyer 1999; Haisch, Lada, \& Lada 2001). The samples of low-mass stars surveyed are dominated by embedded associations with ages of $<3 \mathrm{Myr}$ (e.g. TauAur, Cha I, etc.), and older open clusters with ages of 30-100 Myr (e.g. Pleiades, IC 2602, $\alpha$ Per, etc.). Few well-studied pre-MS stars of 3-30 Myr-old ages have been surveyed. The situation has recently been slightly ameliorated by the discoveries of the TW Hya association and $\eta$ Cha cluster (Kastner et al. 1997; Webb et al. 1999; Mamajek et al. 1999). Yet these samples are small ( 10-20 stars) and dominated by K and Mtype stars with masses of 0.1-0.8 $M_{\odot}$. Our pre-MS star sample is unique in its mass $\left(\sim 1-1.5 M_{\odot}\right)$ and age range $(\sim 10-20 \mathrm{Myr})$, so measuring its disk- 
frequency provides a valuable datum.

Stars with $\mathrm{EW}(\mathrm{H} \alpha)>10 \AA$ in emission are usually called Classical T Tauri stars (CTTSs), which show spectroscopic signatures of accretion as well as near-IR excesses (e.g. Hartigan, Edwards, \& Ghandour 1995). Stars lacking the strong $\mathrm{H} \alpha$ emission and near-IR excesses are called Weaklined T Tauri stars (WTTSs). This can be explained as a correlation between the presence of magnetospheric accretion columns and an inner accretion disk (e.g. Meyer, Calvet, \& Hillenbrand 1997; Muzerolle, Hartmann, \& Calvet 1998). Our $\mathrm{H} \alpha \mathrm{EW}$ measurements are discussed in $\S 4.2 .1$, and here we quantify the K-band excess of our targets. We calculate the intrinsic $J-K$ color excess $\mathrm{E}(J-K)$ o as defined by Meyer, Calvet, \& Hillenbrand (1997):

$E(J-K)_{\circ}=(J-K)_{o b s}-(J-K)_{\circ}-A_{V} \times\left(A_{J}-A_{K}\right)$

where $(J-K)_{o b s}$ is the observed color and $J-K_{\circ}$ is the intrinsic color of an unreddened dwarf star of appropriate spectral type (Kenyon \& Hartmann 1995). Uncertainties in each quantity were propagated in order to estimate the signal-to-noise of the intrinsic color excess. The distribution of measured $\mathrm{E}(J-K)$ 。 values indicate a systematic offset of a few hundredths of a mag. Our near-IR data set is from the 2MASS working database, so we suspect that the absolute calibration or uncertainties in color correction could be responsible and apply a small color correction to account for it. After the correction, the distribution of $\mathrm{E}(J-K)$ 。 values is symmetric about zero, with a few positive and negative $\sim 2 \sigma$ points. There is only one star with a $\mathrm{E}(J-K)$ 。 color excess with $\mathrm{S} / \mathrm{N}>2.5$ : star \#34 = TYC 9246-971-1 has an intrinsic color excess of $\mathrm{E}(J-K)_{\circ}=0.26 \pm 0.06$ implying a $\mathrm{K}$ band excess. This star also happens to be the only CTT identified in our optical spectra $(\mathrm{EW}(\mathrm{H} \alpha)=$ -39 A). TYC 9246-971-1 (= PDS 66, Hen 3-892) was originally identified as an emission line star by Henize (1976), and classified as a CTT in the Pico dos Dias survey of stars in the IRAS PSC catalog (Gregorio-Hetem et al. 1992). By virtue of its position, proper motion, and spectral characteristics, we find that TYC 9246-971-1 is a $\approx 8$ Myr-old, $\approx 1.2 M_{\odot}$ (DM97 tracks) member of the LCC subgroup. Our secular parallax for TYC 9246-971-1 yields a distance of $86_{-7}^{+8} \mathrm{pc}$; the third nearest of the LCC pre-MS stars in our sample, and among the nearest CTTSs known. Only $1 / 58\left(1.7_{-1.4}^{+4.0} \%\right.$; $1 \sigma$ Poisson) of pre-MS stars in LCC are classified as bona fide CTTS, along with none $(0 / 42)$ of the pre-MS members of UCL. For our accretion disk frequency statistics, we use the isochronal ages derived from the DM97 evolutionary tracks (which agree well with the turn-off ages), and include the entire sample of 110 pre-MS stars (including the cooler stars which bias the mean to younger ages). Only $1 / 110\left(0.9_{-0.8}^{+2.1} \% ; 1 \sigma\right)$ of $1.3 \pm 0.2(1 \sigma) M_{\odot}$ stars with ages of $13 \pm 1$ (s.e.) $\pm 6(1 \sigma)$ Myr are CTTSs. This implies that accretion terminates in solar-type stars within the first $15 \mathrm{Myr}$ of their evolution.

\section{Discussion}

We can address several interesting questions regarding the star-formation history of Sco-Cen with data from our survey. Could the Sco-Cen progenitor giant molecular cloud (GMC) have produced a substantial population of low-mass stars for an extended period (>5-10 Myr) before conditions were right to form an OB population? Conversely, is there evidence for any low-mass star-formation after the bulk of the high-mass OB stars formed? The OB star-formation in LCC and UCL has apparently destroyed the progenitor GMC through supernovae and stellar winds (e.g. de Geus 1992; Preibisch \& Zinnecker 2000). However the region is not totally devoid of molecular gas (e.g. the Lupus complex). We will first examine whether there is any evidence of star-formation prior to the formation of the OB subgroups ( $\$ 8.1$ ), and then assess the evidence for more recent star-formation in the UCL region (\$8.2). We will address the age of LCC in $\S 8.3$, and discuss the formation of the subgroups in $\$ 8.4$ Throughout the discussion, we adopt the DM97 ages, as they agree more closely with the turn-off ages than do the SDF00 and PS01 ages.

\subsection{Is There Evidence for Star-Formation Before the Primary Bursts?}

Is our survey sensitive to older stars which may have preceded the primary star-formation episode? Three pre-MS stars in our sample have isochronal ages of $>25 \mathrm{Myr}$ (or undefined as lying 
below the ZAMS), however given the uncertainties in $T_{\text {eff }}$ and $\log \mathrm{L} / \mathrm{L}_{\odot}$ (Fig. 5), even a coeval $\approx 15$ Myr-old population would be expected to have statistical outliers. Here we explore three possible ways in which older ZAMS stars could have escaped our attention.

One could argue that our surface gravity indicator is biasing our sample against identifying ZAMS stars members (if they exist). If we disregard surface gravity as a criterion, we gain only 4 more RASS-ACT/TRC stars (all between F8.5 and G1), and only one of those would have an isochronal age $>25 \mathrm{Myr}$ (TYC 8222-105-1; 30 $\mathrm{Myr}$ ). If they were legitimate, older members with real ages of $>25 \mathrm{Myr}$, they should also be among the stars with the oldest isochronal ages in our sample, which they are not. This suggests that their secular parallaxes, hence their luminosities, are unjustified, and that they are not members of the OB subgroups. Coincidently, TYC 8222-1051 is one of the earliest type stars in our sample (F8.5), where our surface gravity indicator has the least fidelity (Fig. 1). We can state that only one of the Li-rich stars showing dwarf gravity signatures that is co-moving with LCC and UCL has an H-R diagram position and gravity suggestive of ZAMS status.

If a significant ZAMS population existed in LCC and UCL, would our magnitude and X-ray flux limits allowed their detection? X-ray surveys of the ZAMS-age clusters IC 2602 and IC 2391 ( $\sim 30-50 \mathrm{Myr})$ by Randich et al. (1995) and Patten \& Simon (1996) found that late-F and early$\mathrm{G}$ stars ZAMS stars have X-ray luminosities of $\mathrm{L}_{X} \simeq 10^{29.0}-10^{30.5} \mathrm{erg} \mathrm{s}^{-1}$, with $\mathrm{L}_{X} / \mathrm{L}_{b o l}$ ranging from $10^{-3.0}$ to $10^{-4.8}$. The X-ray and optical flux limits imposed by the ROSAT All-Sky Survey and the Tycho catalog allow us to detect ZAMS sources with $\mathrm{L}_{X} / \mathrm{L}_{b o l}>10^{-3.2}$ within $140 \mathrm{pc}$ if they exist. If we adopt the X-ray luminosities of the G stars in IC 2602 and IC 2391 as representative for a $\sim 30$ Myr-old population, we should have detected roughly one-third of a putative ScoCen ZAMS population between masses of 1 and $1.2 M_{\odot}$. We can put a rough upper limit on the number of $>25$ Myr-old stars in our mass range. Assuming that TYC 8222-105-1 is a ZAMS member, and that its H-R diagram position is not a statistical fluctuation from the locus of 15 Myrold stars, we detect one ZAMS star with age $>25$
Myr in the mass range $\left(1-1.2 M_{\odot}\right)$. Accounting for the two-thirds of the ZAMS stars which would have undetectable X-ray emission, and extrapolating over a Kroupa (2001) IMF, this implies a population of $\sim 100$ stars with masses greater than $0.1 M_{\odot}$. This is $\leq 10 \%$ of the stellar population predicted to exist in each OB subgroup $(\sim 1000-$ $2000 ; \S 5.3)$.

Could such ZAMS stars have left the region we probed? If we postulate that the population was very centrally concentrated and gravitationally bound until the OB stars destroyed the giant molecular cloud (GMC) some $10 \mathrm{Myr}$ ago (de Geus 1992), then a $2 \mathrm{~km} \mathrm{~s}^{-1}$ motion radially away from the subgroup center would have moved the star $20 \mathrm{pc}$ in the past $10 \mathrm{Myr}$. This distance is the approximate radius of both of the subgroups today (see Fig. 9 of dZ99). Hence if an older population was concentrated at center of the gravitationallybound GMC until the high mass stars destroyed the cloud, we would find them within the projected boundaries of the subgroups so long as they inherited velocities of $<2 \mathrm{~km} \mathrm{~s}^{-1}$. The kinematic selection procedure of Hoogerwerf (2000) would have selected such stars, since a large velocity dispersion $\left(3 \mathrm{~km} \mathrm{~s}^{-1}\right)$ was initially assumed.

We conclude that there is no evidence for significant star-formation in the LCC and UCL progenitor giant molecular clouds before the primary starformation episodes. Our findings are consistent with the idea that molecular clouds form stars over a range of masses, and dissipate within timescales of $\sim 10 \mathrm{Myr}$.

\subsection{On-going Star-Formation?}

Two obvious sources of young stars may be contaminating the UCL pre-MS sample. The youngest, unembedded OB subgroup of Sco-Cen is US, with a nuclear age of 5-6 Myr (de Geus et al. 1989). US borders UCL near Galactic longitude $343^{\circ}$, and its space motion and distance are very similar to that of UCL (dZ99). The Lupus molecular clouds are also in the western region of UCL (roughly between $335^{\circ}<\ell<345^{\circ}$ and $+5^{\circ}$ $<b<25^{\circ}$ ). The $\mathrm{T}$ Tauri star population within the major Lupus clouds was surveyed by Hughes et al. (1994), and the region was recently mapped in ${ }^{12} \mathrm{CO}$ by Tachihara et al. (2002). Dozens of premain sequence stars were identified outside of the main cores by a pointed ROSAT survey (Kraut- 
ter et al. 1997), and the All-Sky Survey (Wichmann et al. 1997b). The clouds lie at $d=140 \mathrm{pc}$ (Hughes, Hartigan, \& Clampitt 1993), situated spatially between the US and UCL subgroups of Sco-Cen (both $d \simeq 145 \mathrm{pc}$ ). Fig. 10 illustrates the positions of the primary Lupus clouds, the preROSAT Lupus $\mathrm{T}$ Tauri star population, the preMS stars from our survey, and the B-star population of the OB subgroups.

How does the presence of US and the Lupus molecular clouds (and their associated $\mathrm{T}$ Tauri stars) affect our findings regarding the mean age of the UCL subgroup? We split our unbiased $\left(\log \mathrm{T}_{\text {eff }}>3.73\right)$ "pre-MS" and "pre-MS?" members of UCL into two groups using the Galactic longitude line $335^{\circ}$ as a division. Most of the molecular cloud mass in the Lupus region lies between $335^{\circ}<\ell<345^{\circ}$ (see Fig. 2 of Tachihara et al. (2002)). Using the DM97 tracks, we find that the "eastern" UCL pre-MS sample surrounding the Lupus clouds has a mean age of $13 \pm 1 \mathrm{Myr}$, while the "western" UCL sample is somewhat older ( $16 \pm 1 \mathrm{Myr})$. The UCL stars with ages of $<10 \mathrm{Myr}$ are found in greater numbers near the Lupus clouds and US border, supporting the idea that our UCL sample is probably contaminated by more recent star-formation. The age estimate of UCL for the stars west of $\ell=335^{\circ}$ is probably more representative of the underlying UCL population.

Three of the youngest stars (HIP 81380, TYC 7858-830-1, and TYC 7871-1282-1; 5-9 Myr; DM97 tracks) in our entire survey are positioned near a clump of $8 \mathrm{~B}$ stars at $(\ell, b)=\left(343^{\circ},+4^{\circ}\right)$. These three pre-MS stars also have secular parallax distances of $\sim 200 \mathrm{pc}$, similar to what de Bruijne (1999b) found for the group of B stars. The secular parallaxes may be biased, however, if this clump has slightly different kinematics than the average UCL motion. This clump may represent substructure within UCL. However de Bruijne (1999b) was unable to demonstrate that the clump had distinct kinematics or age. The mean Hipparcos distance of the clump B stars is $175 \mathrm{pc}$, with HIP $82514\left(\mu^{1}\right.$ Sco; B1.5Vp) and HIP $82545\left(\mu^{2}\right.$ Sco; B2IV) as the most massive members. Our identification of 3 new pre-MS stars in the same region with similar secular parallaxes supports the notion that this may be a separate subgroup.

Some of our pre-MS stars were also identified in the ROSAT surveys of the Lupus region by Krautter et al. (1997) and Wichmann et al. (1997b) (see notes in Table 6). The presense of a significant population of pre-MS stars outside of star-forming molecular clouds has been attributed by various authors to be due to one or more of the following: (1) slow diffusion (1-2 $\mathrm{km} \mathrm{s}^{-1}$ ) from existing molecular clouds (e.g. Wichmann et al. 1997a), (2) ejection from small-N body interactions (Sterzik \& Durisen 1995), (3) formation in situ from shortlived cloudlets (Feigelson 1996), or (4) fossil starformation associated with the Gould Belt (e.g. Guillout et al. 1998). Wichmann convincingly showed that most of the young RASS stars in the Lupus region are at a distance of around $\sim 150 \mathrm{pc}$ (similar to previously published distances for the Lupus clouds and UCL), and that the stars are roughly 10 Myr-old. Wichmann concludes that the dispersed pre-MS population is most likely a manifestation of the Gould Belt. The OB subgroups of Sco-Cen are major sub-structures of the Gould Belt (as defined by age and kinematics; Frogel \& Stothers 1977), i.e. UCL is the dominant Gould Belt substructure in the Lupus region. We interpret the presence of dozens of pre-MS stars near the Lupus clouds to be primarily the lowmass membership of the UCL OB subgroup. Our analysis suggests that younger US or Lupus stars are a minor contaminant to our UCL sample.

\subsection{Is LCC Older than UCL?}

Although our pre-MS and turn-off age estimates for LCC agree rather well, they are substantially older (by $\sim 50 \%$ ) than previous values. The de Geus et al. (1989) age estimate (11-12 Myr) appears to hinge primarily on the H-R diagram position of $\epsilon$ Cen, with $\delta \mathrm{Cru}, \alpha$ Mus, and $\xi^{2}$ Cen defining the rest of the turn-off isochrone. The latter three stars were confirmed as members by dZ99, however $\epsilon$ Cen (the most massive) was rejected. Although our age for $\epsilon$ Cen is consistent with de Geus's, we omitted it from our LCC age estimate. If one uses the long-baseline proper motion for $\epsilon$ Cen from the new Proper Motions of Fundamental Stars (PMFS) catalog (Gontcharov et al. 2001), and adopt the LCC space motion, convergent point, and formulae of dZ99 (with $\left.v_{\text {int }}=3 \mathrm{~km} \mathrm{~s}^{-1}\right), \epsilon$ Cen has a $100 \%$ membership probability. The resulting secular parallax $\left(\pi_{\text {sec }}=9.6 \pm 2.0\right.$ mas $)$ agrees well with the Hippar- 
$\cos$ astrometric parallax $\left(\pi_{H I P}=8.7 \pm 0.8\right.$ mas $)$, further strengthening the interpretation that $\epsilon$ Cen is a bona fide LCC member. Including $\epsilon$ Cen with the other 5 turn-off stars discussed in $\S 7.2$ does not change our turn-off age estimate, however $(16 \pm 1 \mathrm{Myr})$. If one ignores the stars with masses less than that of $\epsilon$ Cen, then the 12-Myr Bertelli isochrone would appear to be an acceptable fit for LCC. Because the turn-off is poorly defined, we give equal weight to the next five Hipparcos members down the mass spectrum $(\delta \mathrm{Cru}$, $\sigma$ Cen, $\alpha$ Mus, $\mu^{1}$ Cru, and $\xi^{2}$ Cen), which yields an age older than de Geus's.

$\epsilon$ Cen is one of several "classical" LCC early Btype member candidates rejected as members of Sco-Cen using the Hipparcos astrometry. These stars have been included in Sco-Cen candidate membership lists on and off over the past halfcentury: HIP 59196 ( $\delta$ Cen; B2IVne), HIP 60718A ( $\alpha^{1}$ Cru A; B0.5IV), HIP 62434 ( $\beta$ Cru; B0.5IV), and HIP 68702 ( $\beta$ Cen; B1III). These stars are $\sim 10-20 M_{\odot}$ star, with inferred ages of $\sim 5-15 \mathrm{Myr}$, and distances of $\sim 100-150 \mathrm{pc}$. Such stars are extremely rare, and their presense in the LCC region appears to be more than coincidental. Are they all LCC members whose Hipparcos proper motions are perturbed due to binarity? All five systems are flagged (field \#59) in the Hipparcos catalog as stars with unusual motions due to either unseen companions or variability. A kinematic investigation of these stars, and their potential membership in LCC is beyond the scope of this study, but necessary for understanding the global starformation history of the Sco-Cen region. Are these stars bona fide members? If so, why are they so much younger than the other members (both preMS and mid-B stars)? If they are not bona fide members, where did they come from? Although our age estimates for the pre-MS sample and Hipparcos early-B members appear to be consistent, the presense of these young, B0-B2 classical members (Hipparcos non-members) hints that the story of star-formation in Sco-Cen is more complex than our results reveal.

\subsection{A Star-Formation History of Sco- Cen?}

Preibisch \& Zinnecker (2000) reviewed the recent star-formation history of Sco-Cen $(<5-10$ Myr) in the region of US, UCL, and $\rho$ Oph. They present evidence for external supernovae triggering in the formation of the subgroups. They claim that supernovae shock waves from UCL passed through the US progentior GMC approximately 5 Myr ago, and caused the cloud to collapse. The US group appears to have had at least one supernova in the past $\sim 1$ Myr, possibly a deceased massive companion to the runaway $09.5 \mathrm{~V}$ star $\zeta$ Oph. This supernova contributed to destroying the GMC and producing the US superbubble (de Geus 1992; Hoogerwerf, de Bruijne, \& de Zeeuw 2001). The US subgroup appears to be currently triggering star-formation in the $\rho$ Oph cloud core. Here we speculate on the global star-formation history of the Sco-Cen complex.

The formation of LCC and/or UCL may have been similarly triggered. However it is unclear if one triggered the formation of the other or vice versa. Our pre-MS age estimates are consistent with LCC being slightly older than UCL by a few Myr, though they could be coeval. What was the origin of these large OB subgroups? The gas associated with the Sco-Cen complex appears to be part of the Lindblad Ring, a torus of $\mathrm{H} \mathrm{I}$ and molecular clouds hundreds of pc in radius. It is centered roughly near the $\alpha$ Persei cluster and Cas-Tau OB association (Blaauw 1991; Pöppel 1997). The young stars that have formed from this gas complex (i.e. the Gould Belt) share a systematic expansion consistent with a localized origin for the whole complex - probably an expanding gas shell from a large star-formation event (e.g. Moreno, Alfaro, \& Franco 1999). The gas associated with Sco-Cen appears to be part of a "spur" of neutral hydrogen and molecular clouds that runs from near LCC (including Coalsack, Musca, and Chamaeleon clouds), through Lupus, Ophiuchus, and into the Aquila and Vulpecula Rift regions (see Fig. 3-18 of Pöppel 1997). It is likely that LCC and UCL were among the first clumps in the Lindblad Ring to collapse and form stars (see $\S 4$ of Blaauw 1991), either from self-gravity or from triggered from external supernovae events. The LCC and UCL regions formed a large population of $\mathrm{OB}$ stars and their stellar winds and supernovae may indeed have triggered the collapse of the US group. The process might continue over the next 10 Myr as the supernovae from the US and $\rho$ Oph subgroups send shock waves into the vast reservoir of atomic and molecular gas asso- 
ciated with the Aquila Rift (see $\$ 3.4$ of Pöppel 1997, and references therein). On the other side of Sco-Cen, there appears to be little gas westward of LCC until one reaches the Vela complex some $400 \mathrm{pc}$ away. The lack of a sufficient gas reservoir probably explains why triggering did not proceed to form OB subgroups west of LCC.

The Sco-Cen subgroups have formed their own network of superbubbles with radii of $\sim 100 \mathrm{pc}$ (de Geus 1992). The superbubbles appear to be largely H I, presumably gas from the progenitor Sco-Cen GMC as well as the swept-up interstellar medium. In some regions, they are associated with well-known nearby molecular cloud complexes: Coalsack, Musca, Chamaeleon, Corona Australis, Lupus, and numerous small high Galactic latitude clouds (e.g. Bhatt 2000). The Lupus clouds are spatially coincident with the western side of the US superbubble, however no kinematic analysis has been yet undertaken to determine whether the Lupus clouds share in the bubble expansion. The CrA molecular clouds are embedded within the UCL superbubble shell, and the space motion of the $\mathrm{T}$ Tauri star population is moving radially away from UCL (Mamajek \& Feigelson 2001). Other young stars in the field toward the 4th Galactic quadrant, including the $\eta$ Cha cluster, TW Hya association, and $\beta$ Pic group, all have ages of $\sim 10 \mathrm{Myr}$ and are moving radially away from LCC and UCL. Perhaps these stars formed in small molecular clouds that accumulated within the expanding LCC/UCL superbubble shells.

\section{CONCLUSIONS}

From our spectroscopic survey of an X-ray- and kinematically-selected sample of late-type stars in the Sco-Cen OB association, we summarize our main findings as follows:

- We have identified a population of low-mass stars in the Lower Centaurus-Crux (LCC) and Upper Centaurus-Lupus (UCL) OB subgroups with the following properties: (1) G$\mathrm{K}$ spectral types, (2) subgiant surface gravities, (3) Lithium-rich, (4) strong X-ray emission $\left(\mathrm{L}_{X} \simeq 10^{30}-10^{31} \mathrm{erg} \mathrm{s}^{-1}\right)$, and (5) proper motions consistent with the highmass members. We classify stars which show these characteristics as bona fide pre-MS stars or "post-T Tauri" stars. X-ray and kinematic selection (the RASS-ACT/TRC sample) yielded a hit rate of $93 \%$ for selecting probable pre-MS stars, while kinematic selection alone (dZ99 Hipparcos sample G-K dwarfs and subgiants) yielded $73 \%$.

- We estimate the mean age of the preMS population in the LCC subgroup to be $17 \pm 1 \mathrm{Myr}$ (DM97 tracks), $21 \pm 2 \mathrm{Myr}$ (PS01), and 23 $\pm 2 \mathrm{Myr}$ (SDF00). For UCL, the pre-MS population's mean age is $15 \pm 1 \mathrm{Myr}$ (DM97), $19 \pm 1 \mathrm{Myr}$ (PS01), and $22 \pm 1 \mathrm{Myr}$ (SDF00). The UCL pre-MS estimate appears to be slightly biased towards younger ages (by $\sim 1 \mathrm{Myr}$ ) through contamination by Lupus or US members. We also calculate new MS turn-off ages of $16 \pm 1 \mathrm{Myr}$ for LCC and $17 \pm 1 \mathrm{Myr}$ for UCL using the dZ99 Hipparcos membership and Bertelli et al. (1994) evolutionary tracks. The UCL pre-MS and turn-off age estimates are roughly self-consistent, and similar to previously published estimates. Our age estimates for LCC (pre-MS and turn-off) are older than previous estimates, and are equal to or slightly older than UCL.

- We find that $68 \%$ of the low-mass starformation in each subgroup took place within a <4-6 Myr span, and 95\% took place within <8-12 Myr (using DM97 tracks). The conditions were right for producing low-mass stars in the LCC and UCL progenitor molecular clouds for $<10$ Myr.

- We find the frequency of CTTSs among a pre-MS population in an OB association with masses of $1.3 \pm 0.2(1 \sigma) M_{\odot}$ and ages of $13 \pm 1$ (s.e.) $\pm 6(1 \sigma)$ Myr (DM97 tracks) to be only $0.9_{-0.8}^{+2.1 \%}(1 / 110)$. The younger age results from using our entire (i.e. magnitude-biased) sample of pre-MS stars. Only one star in our sample showed both strong $\mathrm{H} \alpha$-emission and a $\mathrm{K}$-band excess: the previously known CTTS TYC 9246-971-1 (\#34 = PDS $66=$ Hen 3-892). This suggests that the disk accretion phase lasts $\leq 10-20 \mathrm{Myr}$ in the evolution of solartype stars in OB associations.

- We demonstrate that a surface gravity indicator for classifying field $\mathrm{G}$ and $\mathrm{K}$ stars 
(Sr II $\lambda 4077$ to Fe I $\lambda 4071$ ) can be used to distinguish whether Li-rich stars are pre-MS or ZAMS in nature. When this indicator is used in tandem with other youth diagnostics (Li abundance, X-ray emission, $\mathrm{H} \alpha$ emission, and kinematics), one can confidently classify a star as pre-MS in nature.

EM and MM acknowledge support through NASA contract 1224768 administered by JPL. EM and JL were also supported through a NASA/JPL grant. EM also thanks the $\Sigma \Xi$ Research Honor Society for a Grant-in-Aid which financed part of our observing run. We thank the TAC of the Mt. Stromlo and Siding Springs Observatories 2.3-metre telescope for their generous allocation of telescope time. This project benefited from discussions and help from J. Beiging, A. Blaauw, J. Carpenter, C. Corbally, J. de Bruijne, T. de Zeeuw, M. Hamuy, L. Hillenbrand, W. Lawson, D. Soderblom, J. Stauffer, and S. Strom. We offer special thanks to Ronnie Hoogerwerf for assistance in the target selection effort and discussions. This publication makes use of data products from the Two Micron All Sky Survey (2MASS), which is a joint project of the Univ. of Massachusetts and the Infrared Processing and Analysis Center (IPAC), funded by NASA and NSF. 


\section{A. The Pre-Main Sequence $T_{\text {eff }}$ Scale}

Pre-main sequence stars lie between dwarfs (V) and subgiants (IV) on color-absolute magnitude or temperature-luminosity H-R diagrams. A given visual spectral type will correspond to cooler temperatures as surface gravity decreases (e.g. Gray 1991; de Jager \& Nieuwenhuijzen 1987). Dwarf temperature scales are often adopted for pre-MS populations, however it is prudent to account for the effects of surface gravity.

We quantify the effects of $\log g$ on the $\mathrm{SpT}$ vs. $T_{\text {eff }}$ relation using two datasets. First, we fit a polynomial surface to $T_{\text {eff }}(\mathrm{SpT}, \log g)$ using the data from Gray (1991, Table 2). As with most compilations of $T_{\text {eff }}(\mathrm{SpT})$ in the FGK-star regime, we find that a trinomial is the best low-order fit, and that a linear dependence on $\log g$ adequately accounts for the effects of surface gravity on temperature. Our second method finds a similar surface fit to $T_{\text {eff }}(\mathrm{SpT}, \log g)$ using published $T_{\text {eff }}$ and $\log g$ values (Cayrel de Strobel et al. 2001) for the GK standards of Keenan \& McNeil (1989) and F standards of Garcia (1989) (those within 0.3 dex of solar $[\mathrm{Fe} / \mathrm{H}]$, and luminosity class IV and $\mathrm{V}$ only). We adopt the isochrones from D'Antona \& Mazzitelli (1997) for a fiducial $\log g$ value as a function of $T_{\text {eff }}$ for a coeval 15-Myr-old population.

Both assessments yield essentially the same result: dwarf temperature scales for G-K stars should be lowered by $35 \mathrm{~K}$ for a $15 \mathrm{Myr}-\mathrm{old}$ population. The temperature decrement increases for younger ages: $70 \mathrm{~K}-40 \mathrm{~K}$ for G0-K2 10-Myr-old stars, $235 \mathrm{~K}-105 \mathrm{~K}$ for G0-K2 5-Myr-old stars, and $260 \mathrm{~K}-180 \mathrm{~K}$ for G3-K2 1-Myr-old stars. Both techniques yielded a linear dependence of $\log g$ on $T_{\text {eff }}(\mathrm{SpT}, \log g$ ), and the slopes were similar: $\partial T_{e f f} / \partial \log g \simeq 220 \mathrm{~K} / \log \left(\mathrm{cm} \mathrm{s}^{-1}\right)$ for the Keenan standards with Cayrel de Strobel stellar atmosphere data, and $\partial T_{\text {eff }} / \partial \log g \simeq 190 \mathrm{~K} / \log \left(\mathrm{cm} \mathrm{s}^{-1}\right)$ for the interpolation of Gray's (1991) Table 2 . As the evolutionary model isochrones are parallel to the main sequence when the stars are on the radiative tracks (i.e. $\sim 10-30 \mathrm{Myr}$ for $\sim 1 M_{\odot}$ stars), the $\Delta \log g$ between a $15 \mathrm{Myr}$-isochrone and the main sequence is fairly constant over the G-K spectral types. Hence one naively expects a linear offset in $T_{\text {eff }}(\operatorname{SpT}, \log g)$ between the 15-Myr isochrone and the MS.

With the $1 \sigma$ scatter between published $T_{\text {eff }}(\mathrm{SpT})$ relations being $\sim 60 \mathrm{~K}$ amongst $\mathrm{G}$ stars, the systematic shift is nearly negligible. Upon comparing several temperature scales from the literature, we adopt the dwarf $T_{\text {eff }}$ scale from Schmidt-Kaler (1982), and apply a $-35 \mathrm{~K}$ offset to correct for the effects of lower surface gravity for a putative 15-Myr-old population. We conclude that adopting dwarf $T_{\text {eff }}$ vs. SpT scales for pre-MS stars younger than $\sim 10 \mathrm{Myr}$ will systematically overestimate their $T_{\text {eff }}$ values, and in turn, their masses inferred from evolutionary tracks. This could have deleterious systematic effects on derived initial mass functions for young associations.

\section{B. Standards with Questionable Luminosity Class}

Several of the standard stars we observed had H-R diagram positions, published $\log g$ values, and Sr II $\lambda 4077 / \mathrm{Fe}$ I $\lambda 4071$ ratios ( $\mathrm{SrFe}$ ) which differed from what is expected for their luminosity classes given in Keenan \& McNeil (1989). The differences are only at the half of a luminosity-class level. We adopt the Keenan temperature types for all of his standard stars, however we revised the luminosity classes of these stars to bring their H-R diagram positions, $\mathrm{SrFe}$ index, and published $\log g$ estimates into harmony (Table 9). The SrFe indices for the vast majority of the standards formed loci according to luminosity class (Fig. 1), so we are comfortable using the index as an additional descriminent. The dwarf regression line in the gravity indicator vs. temperature indicator plot (Fig. 1) was constructed using only Keenan standards for which his luminosity classification agreed with published $\log g$ values and the H-R diagram position. 


\section{Polynomial Fits}

- MI6 vs. Spectral Type: This flux ratio is Index 6 of Malyuto \& Schmidt-Kaler (1997). We measure the index in magnitudes (MI6 $=-2.5 \log (\mathrm{f}(\lambda \lambda 5125-5245) / \mathrm{f}(\lambda \lambda 5245-5290)))$, and find the following relation for Keenan and Garcia F0-K6 III-IV standards (Table 3) within 0.3 dex of solar metallicity:

$$
\begin{gathered}
S p T=33.26 \pm 0.07+(22.75 \pm 0.48) \times M I 6 \quad(0.06<M I 6<0.26) \\
S p T=30.82 \pm 0.16+(105.38 \pm 8.28) \times M I 6-(711.74 \pm 173.81) \times M I 6^{2} \quad(-0.04<M I 6<0.06)
\end{gathered}
$$

where SpT is the spectral type on Keenan's (1984) scale, i.e. F5 $=28$, F8 $=29$, G0 $=30, \mathrm{G} 2=31$, G5 $=$ $32, \mathrm{G} 8=33, \mathrm{~K} 0=34, \mathrm{~K} 1=35$, and $\mathrm{K} 2=36$. Intermediate types can be assigned e.g. G9 = 33.5, G9.5 = $33.75, \mathrm{~K} 0+=34.25, \mathrm{~K} 0.5=34.5$, etc. The first equation applies to K0-K6 stars, and the second equation applies to $\mathrm{F} 0-\mathrm{K} 0$ stars. The $1 \sigma$ dispersion in these fits is 0.6 subtypes.

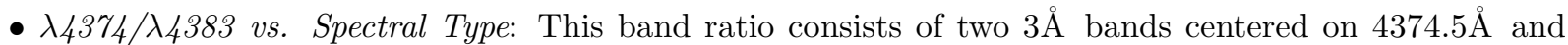

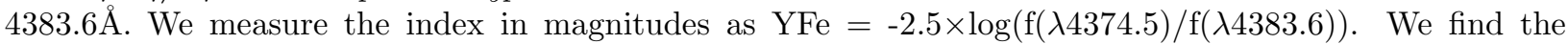
following relation between the band ratio and SpT for F0-K6 III-V stars:

$$
S p T=25.97 \pm 0.30+(20.47 \pm 0.90) \times Y F e
$$

The residual standard deviation to the fit (using 20 Keenan F0-K5 III-V standards within 0.3 dex of solar metallicity) is 0.6 subtypes.

- Surface Gravity Index Fe I $\lambda 4071 / S r$ II $\lambda 4077$ vs. Spectral Type: We measure a surface gravity index using the flux ratio of two $3 \AA$ bands centered on Fe I $\lambda 4071.4$ and Sr II $\lambda 4076.9$. We measure the flux ratio in magnitudes: $\mathrm{SrFe}=-2.5 \times \log (\mathrm{f}(\lambda 4071) / \mathrm{f}(\lambda 4077))$, and plot against our MI6 spectral type index. The Keenan standard dwarfs confirmed as being main sequence stars define a narrow locus:

$$
S r F e=-0.078 \pm 0.005+(2.123 \pm 0.261) \times M I 6-(8.393 \pm 2.945) \times M I 6^{2}+(15.487 \pm 8.672) \times M I 6^{3}
$$

The $1 \sigma$ sample standard deviation of this fit is 0.0094 mag in SrFe. The boundary between dwarfs and subgiants in Fig. 1 is $-2 \sigma$ of the dwarf locus. This relation is valid for F9-K6 stars.

- Ho vs. Spectral Type: In Fig. 2, we fit the equivalent widths of the $\mathrm{H} \alpha$ feature (at low resolution, the photospheric absorption plus the chromospheric emission) as a function of spectral type for F0-K6 dwarf and subgiant standard stars (Table 3) with the following polynomial:

$$
E W(H \alpha)=2.983 \pm 0.066-(0.456 \pm 0.027) \times(S p T-30)+(2.574 \pm 0.378) \times 10^{-2} \times(S p T-30)^{2}
$$

$\mathrm{EW}(\mathrm{H} \alpha)$ is measured in $\AA$. SpT is spectral type on Keenan's scale (as before). The sample standard deviation of the polynomial fit to 11 standards was $0.20 \AA$.

- Converting Tycho B-V to Cousins-Johnson B-V: The Hipparcos catalog gives linear relations between $B_{T}-V_{T}, B-V, V$, and $V_{T}$ for stars of a wide range in spectral types. Bessell (2000) compared the Hipparcos/Tycho photometry and that of the E-region photometric standards, and refined the relations between the two systems. Table 2 of Bessell (2000) gives a standard relation between $B_{T}-V_{T}$, CousinsJohnson $B-V$, and $\left(\mathrm{V}-\mathrm{V}_{T}\right)$ for B-G dwarfs and $\mathrm{K}-\mathrm{M}$ giants. We fit the following relations to Bessell's tables:

$$
\begin{aligned}
& V=V_{T}+9.7 \times 10^{-4}-1.334 \times 10^{-1}\left(B_{T}-V_{T}\right)+5.486 \times 10^{-2}\left(B_{T}-V_{T}\right)^{2}-1.998 \times 10^{-2}\left(B_{T}-V_{T}\right)^{3} \\
& B-V=\left(B_{T}-V_{T}\right)+7.813 \times 10^{-3}\left(B_{T}-V_{T}\right)-1.489 \times 10^{-1}\left(B_{T}-V_{T}\right)^{2}+3.384 \times 10^{-2}\left(B_{T}-V_{T}\right)^{3}
\end{aligned}
$$




$$
B-V=\left(B_{T}-V_{T}\right)-0.006-1.069 \times 10^{-1}\left(B_{T}-V_{T}\right)+1.459 \times 10^{-1}\left(B_{T}-V_{T}\right)^{2}
$$

The $\mathrm{V}\left(V_{T}, B_{T}-V_{T}\right)$ polynomial equation $\mathrm{C} 6$ applies to stars from $-0.25<\left(B_{T}-V_{T}\right)<2.0$ (B-M types). Equation $\mathrm{C} 7$ is for stars with $0.5<\left(B_{T}-V_{T}\right)<2.0$, and equation C8 is for stars with $-0.25<\left(B_{T}-V_{T}\right)<$ 0.5 . We do not quote uncertainties in the polynomial coefficients since the Bessell relations are already smoothed. These equations fit Bessell's standard relations to 1-2 millimagnitudes. 


\section{REFERENCES}

Balona, L. A., 1994, MNRAS, 268, 119

Basri, G., Marcy, G. W., \& Graham, J. R., 1996, ApJ, 458, 600

Bertelli, G., Bressan, A., Chiosi, C., Fagotto, F., \& Nasi, E., 1994, A\&AS, 106, 275

Bertout, C., 1989, ARA\&A, 27, 351

Bessell, M.S., 2000, PASP, 112, 961

Bessell, M.S. \& Brett, J.M., 1988, PASP, 100, 1134

Bessell, M.S., Castelli, F., \& Plez, B., 1998, A\&A, 333,231

Bhatt, H. C., 2000, A\&A, 362, 715

Blaauw, A., 1946, Ph.D. Thesis, Gronningen University

Blaauw, A., 1991, in The Physics of Star Formation and Early Stellar Evolution, NATO ASI Series C, Vol. 342, eds. C.J. Lada \& N.D. Kylafis (Dordrecht: Kluwer), 125

Bouvier, J., Wichmann, R., Grankin, K., Allain, S., Covino, E., Fernandez, M., Martin, E. L., Terranegra, L., Catalano, S., Marilli, E., 1997, A\&A, 318, 495

Carpenter, J.M., 2001, AJ, 121, 2851

Cayrel de Strobel, G., Soubiran, C., Ralite, N., 2001, A\&A, 373, 159

Covino, E., Alcala, J. M., Allain, S., Bouvier, J., Terranegra, L., \& Krautter, J., 1997, A\&A, 328, 187

Crawford, D. L., 1978, AJ, 83, 48

Crawford, D. L. \& Mandwewala, N., 1976, PASP, 88,917

Cutispoto, G., Pastori, L., Pasquini, L., de Medeiros, J. R., Tagliaferri, G., \& Andersen, J., 2002, A\&A, 384, 491

D’Antona, F. \& Mazzitelli, I., 1997, Mem. Soc. Astr. Ital., 68, 807 (DM97)

de Bruijne, J. H. J., 1999a, MNRAS, 306, 381

de Bruijne, J. H. J., 1999b, MNRAS, 310, 585 de Geus, E., de Zeeuw, P. \& Lub, J., 1989, A\&A, 216,44

de Geus, E., 1992, A\&A, 262, 259

de Jager, C. \& Nieuwenhuijzen, H., 1987, A\&A, 177, 217

de Zeeuw, P. T. \& Brand, J., 1985, in Birth and Evolution of Massive Stars and Stellar Groups; Proceedings of the Symposium, Dwingeloo, Netherlands, September 24-26, 1984, (Dordrecht: D. Reidel), 102

de Zeeuw, P. T., Hoogerwerf, R., de Bruijne, J. H. J., Brown, A. G. A., \& Blaauw, A., 1999, AJ, 117, 354 (dZ99)

Drilling, J. S., \& Landolt, A. U., 2000, in Allen's Astrophysical Quantities, 4th. Ed., ed. Arthur C. Cox, (New York: AIP Press, Springer)

European Space Agency, 1997, The Hipparcos and Tycho Catalogues, ESA SP-1200

Feigelson, E. D., 1996, ApJ, 468, 306

Fleming, T. A., Molendi, S., Maccacaro, T., \& Wolter, A., 1995, ApJS, 99, 701

Frogel, J. A., \& Stothers, R., 1977, AJ, 82, 890

Garcia, B., 1989, Bull. Inform. CDS 36, 27

Gehrels, N., 1986, ApJ, 303, 336

Gontcharov, G. A., Andronova, A. A., Titov, O. A., \& Kornilov, E. V., 2001, A\&A, 265, 222

Gray, D. F., 1991, The Observation and Analysis of Stellar Photospheres, 2nd ed., (New York: Cambridge)

Gray, R.O., 2000, A Digital Spectral Classification Atlas, http://nedwww.ipac.caltech.edu/level5/Gray/

Gray, R.O., Graham, P.W., \& Hoyt, S.R., 2001, AJ, 121, 2159

Gregorio-Hetem, J., Lépine, J. R. D., Quast, G. R., Torres, C. A. O., \& de la Reza, R., 1992, AJ, 103, 549

Guillout, P., Sterzik, M. F., Schmitt, J. H. M. M., Motch, C., \& Neuhäuser, R., 1998, A\&A, 337, 113 
Haisch Jr., K. E., Lada, E. A., \& Lada, C. J., 2001, ApJ, 553, L153

Hamuy, M., Suntzeff, N. B., Heathcote, S. R., Walker, A. R., Gigoux, P., \& Phillips, M. M., 1994, PASP, 106, 566

Hartigan, P., Edwards, S., \& Ghandour, L., 1995, ApJ, 452, 736

Hauck, B. \& Mermilliod, M., 1997, A\&AS, 129, 431

Henize, K. G., 1976, ApJS, 30, 491

Herbig, G. H., 1978, in Problems of Physics and Evolution of the Universe, ed. L. V. Mirzoyan (Yervan: Acad. Sci. Armenian SSR), 171

Herbig, G. H., Bell, K. Robin, 1988, Lick Obs. Bulletin No. 1111, (Santa Cruz: Lick Observatory)

Hillenbrand, L. A. \& Meyer, M. R., 1999, BAAS, 195, \#209

Høg, E., Fabricius, C., Makarov, V.V., Urban, S., Corbin, T., Wycoff G., Bastian U., Schwekendiek P., Wicenec A. 2000a, A\&A, 355, L27

Høg, E., Fabricius, C., Makarov, V.V., Bastian, U., Schwekendiek, P., Wicenec, A., Urban, S., Corbin, T., Wycoff, G. 2000b, A\&A, 357, 367

Høg, E., Kuzmin, A., Bastian, U., Fabricius, C., Kuimov, K., Lindegren, L., Makarov, V.V., \& Roeser, S., 1998, A\&A, 335, L65

Hoogerwerf, R. 2000, MNRAS, 313, 43

Hoogerwerf, R. \& Aguilar, L. A., 1999, MNRAS, 306,394

Hoogerwerf, R., de Bruijne, J. H., J., \& de Zeeuw, P. T., 2001, 365, 49

Houk, N., 1978, Michigan Catalogue of TwoDimensional Spectral Types for HD Stars. (Vol. $\left.2,-52^{\circ}<-40^{\circ}\right),($ Ann Arbor: Univ. Michigan)

Houk, N., 1982, Michigan Catalogue of TwoDimensional Spectral Types for HD Stars. (Vol. $\left.3,-40^{\circ}<-26^{\circ}\right),($ Ann Arbor: Univ. Michigan)
Houk, N. \& Cowley, A. P., 1975, Michigan Catalogue of Two-Dimensional Spectral Types for HD Stars. (Vol. 1, $-90^{\circ}<-53^{\circ}$ ), (Ann Arbor: Univ. Michigan)

Houk, N. \& Smith-Moore, M., 1988, Michigan Catalogue of Two-Dimensional Spectral Types for HD Stars. (Vol. 4, $-26^{\circ}<-12^{\circ}$ ), (Ann Arbor: Univ. Michigan)

Houk, N. \& Swift, C., 1999, Michigan Catalog of Two-Dimensional Spectral Types for the HD Stars. (Vol. 5., $-12^{\circ}<+5^{\circ}$ ), (Ann Arbor: Univ. Michigan)

Hughes, J., Hartigan, P., Clampitt, L., 1993, AJ, 105,571

Hughes, J., Hartigan, P., Krautter, J., \& Kelemen, J., 1994, AJ, 108, 1071

Jensen, E., 2001, in Young Stars Near Earth: Progress \& Prospects, eds. R. Jayawardhana \& T. Greene, ASP Conf. Ser. Vol. 244, (San Francisco: ASP), 3

Johnson, H. L, \& Morgan, W. W., 1953, ApJ, 117, 313

Jones, B. F., Fischer, D., Shetrone, M., \& Soderblom, D. R., 1997, AJ, 114, 352

Kastner, J. H., Zuckerman, B., Weintraub, D. A., \& Forveille, T., 1997, Science, 227, 67

Keenan, P.C., 1984, in The MK Process and Stellar Classification, ed. R. F. Garrison, (Toronto: Univ. of Toronto), 29

Keenan, P.C. \& Barnbaum, C., 1999, ApJ, 518, 859

Keenan, P.C. \& McNeil, R.C., 1989, ApJS, 71, 245

Keenan, P.C. \& McNeil, R.C. 1976, An Atlas of Spectra of the Cooler Stars, (Columbus: Ohio State Univ.)

Keenan, P.C. \& Yorka, S.B., 1988, Bull. Inform. CDS 35, 37

Kenyon, S.J. \& Hartmann, L., 1995, ApJS, 101, 117

Köhler, R., Kunkel, M., Leinert, C., \& Zinnecker, H., 2000, A\&A, 356, 541 
Krautter, J., Wichmann, R., Schmitt, J.H.M.M., Alcala, J.M., Neuhauser, R., \& Terranegra, L., 1997, A\&AS, 123, 329

Kroupa, P., 2001, MNRAS, 322, 231

Lowrance, P. J., Schneider, G., Kirkpatrick, J. D., Becklin, E. E., Weinberger, A. J., Zuckerman, B., Plait, P., Malmuth, E. M., Heap, S. R., Schultz, A., Smith, B. A., Terrile, R. J., \& Hines, D. C., 2000, ApJ, 541, 390

Maeder, A., 1981, A\&A, 101, 385

Malyuto, V. \& Schmidt-Kaler, Th. 1997, A\&A, 693,699

Mamajek, E.E., Lawson, W.A. \& Feigelson, E.D. 1999, ApJ, 516, L77

Mamajek, E.E., Feigelson, E.D., 2001, Young Stars Near Earth: Progress \& Prospects, ASP Conf. Ser. Vol. 244, eds. R. Jayawardhana \& T. Greene, (San Francisco: ASP), 104.

Mashonkina, L. \& Gehren, T., 2001, A\&A, 376, 232

Mathis, J. S., 1990, ARA\&A, 28, 37

Meyer, M. R., Calvet, N., \& Hillenbrand, L. A., 1997, AJ, 114, 288

Moreno, E., Alfaro, E. J., \& Franco, J., 1999, ApJ, 522,276

Muzerolle, J., Hartmann, L., \& Calvet, N., 1998, AJ, 116, 455

Napiwotzki, R., Schönberner, D., \& Wenske, V., 1993, A\&A, 268, 653

Palla, F., \& Stahler, S. W., 2001, ApJ, 553, 299 (PS01)

Pallavicini, R., Cerruti-Sola, M., \& Duncan, D.K., 1987, A\&A, 174, 116

Patten, B. H., \& Simon, T., 1996, ApJS, 106, 489

Pojmanski, G., 1998, Acta Astron., 48, 35

Pöppel, W., 1997, Fund. Cosm. Phys., 18, 1

Popper, D. M., 1966, AJ, 71, 175

Preibisch, T. \& Zinnecker, H., 1999, AJ, 117, 2381
Preibisch, T. \& Zinnecker, H., 2000, in Stellar Clusters and Associations: Convection, Rotation, and Dynamos, ASP Conf., Vol.198., eds. by R. Pallavicini, G. Micela, and S. Sciortino, 219

Randich, S., Schmitt, J. H. M. M., Prosser, C. F., \& Stauffer, J. R., 1995, A\&A, 300, 134

Randich, S., Aharpour, N., Pallavicini, R., Prosser, C. F., \& Stauffer, J. R., 1997, A\&A, 323,86

Randich, S., Gratton, R., Pallavicini, R., Pasquini, L., \& Carretta, E., 1999, A\&A, 348, 487

Rebull, L. M., Wolff, S. C., Strom, S. E., \& Makidon, R. B., 2001, ApJL, in press

Rodgers, A. W., Conroy, P., \& Bloxham, G., 1988, PASP, 100, 626

Rose, J.A., 1984, AJ, 89, 1238

Schmidt-Kaler, Th., 1982, in Landolt-Börnstein: Numerical Data and Functional Relationships in Science and Technology, eds. K. Schaífers \& H. H. Voigt, (Berlin: Springer-Verlag)

Shobbrook, R. R., 1983, MNRAS, 205, 1215

Siess, L., Dufour, E. \& Forestini, M., 2000, A\&A, 358, 593 (SDF00)

Slawson, R. W., Hill, R. J., \& Landstreet, J. D., 1992, ApJS, 82, 117

Smart, W. M., 1968, Stellar Kinematics, (London:Longmans)

Soderblom, D. R., Jones, B. F., Balachandran, S., Stauffer, J. R., Duncan, D. K., Fedele, S. B., \& Hudon, J. D., 1993, AJ, 106, 1059

Soderblom, D. R., King, J. R., \& Henry, T. J., 1998, AJ, 116, 396

Spangler, C., Sargent, A. I., Silverstone, M. D., Becklin, E. E., Zuckerman, B., 2001, ApJ, 555, 932

Stauffer, J. R., Caillault, J.-P., Gagné, M., Prosser, C. F., \& Hartmann, L. W., 1994, ApJS, 91, 625

Sterzik, M. F., \& Durisen, R. H., 1995, A\&A, 304, L9 
Tachihara, K., Toyoda, S., Onishi, T., Mizuno, A., Fukui, Y., \& Neuhäuser, R., 2002, PASJ, in press.

Urban, S.E., Corbin, T.E., \& Wycoff, G.L. 1998, AJ, 115, 2161

Voges, W., et al., 1999, A\&A, 349, 389

Wallace, L., Hinkle, K., \& Livingston, W. 1998, An Atlas of the Spectrum of the Solar Photosphere from 13,500 to $28,000 \mathrm{~cm}^{-1}$ (3570 to $7405 \AA$ ), (Tucson: NOAO)

Webb, R., A., Zuckerman, B., Platais, I., Patience, J., White, R. J., Schwartz, M. J., McCarthy, C., 1999, ApJ, 512, L63

Wichmann, R., Krautter, J., Covino, E., Alcalá, J. M., Neuhäuser, R., Schmitt, J. H. M. M., 1997, A\&A, 320, 185

Wichmann, R., Sterzik, M., Krautter, J., Metanomski, A., \& Voges, W., 1997b, A\&A, 326,211

Wilking, B. A., Lada, C. J., \& Young, E. T., 1989, ApJ, 340, 823

This 2-column preprint was prepared with the AAS LATEX macros v5.0. 


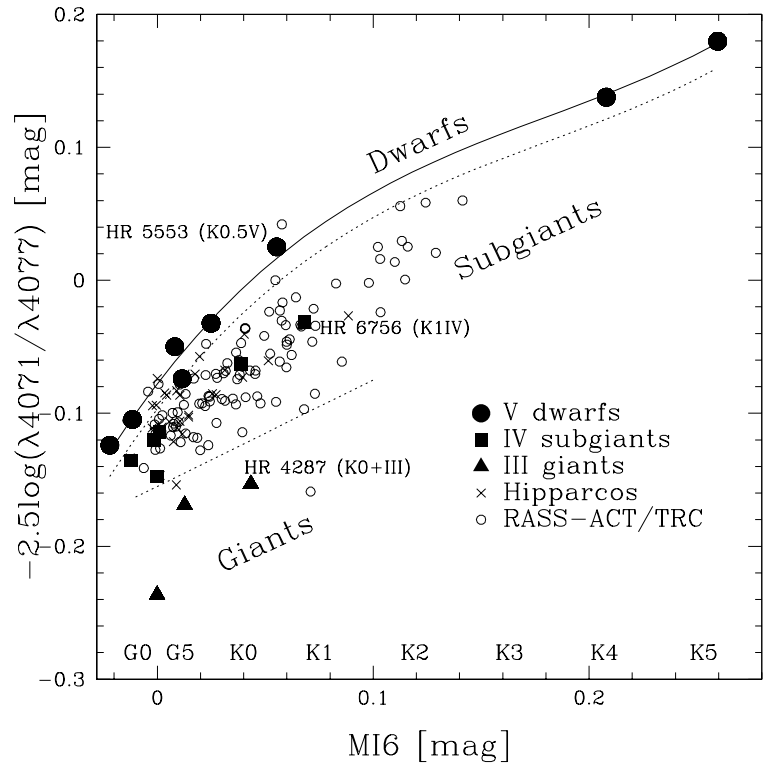

Fig. 1. - The MI6 band-ratio ( $T_{\text {eff }}$ indicator) vs. the band-ratio of Fe I $\lambda 4071 /$ Sr II $\lambda 4077$ (surface gravity indicator). The solid line is a polynomial fit to only the dwarf standards. The dashed lines separate dwarfs, subgiants, and giants. The dwarfsubgiant dashed line is $-2 \times$ the $\sigma$-residual below the dwarf regression, whereas the subgiant-giant boundary is placed somewhat arbitrarily to resolve the observed subgiant and giant loci. Empirically, this diagram suggests that most of the target stars are consistent with being $\mathrm{G}$ and K-type subgiants, with few giant and dwarf interlopers. A few early$\mathrm{K}$ standards are noted for reference.

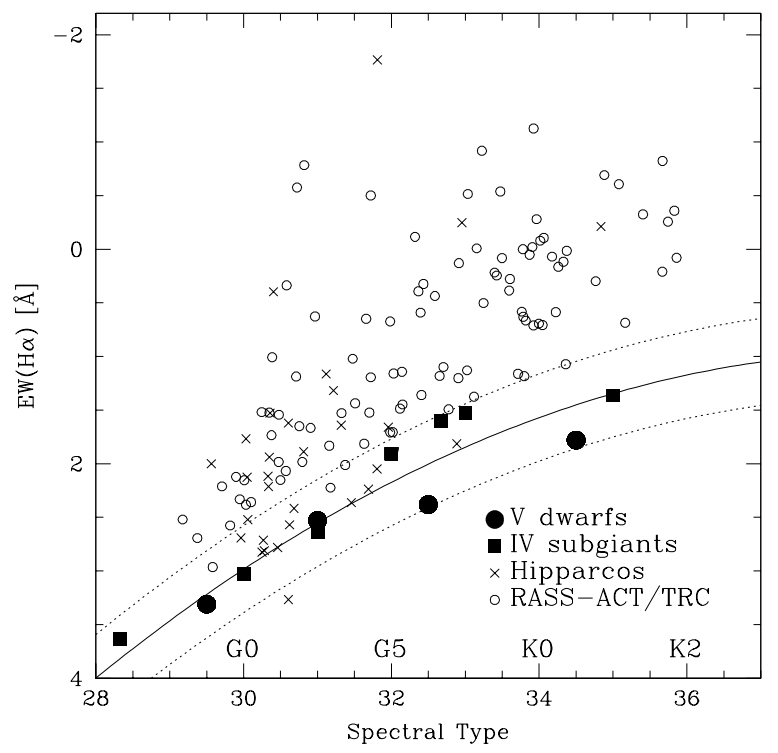

Fig. 2.- $\mathrm{H} \alpha$ EWs for the pre-MS candidates compared to inactive field dwarfs and subgiants. Symbols are the same as for Fig. 1. The solid line is the average $\mathrm{EW}(\mathrm{H} \alpha)$ for dwarf and subgiant standard stars. The dashed line represents the $\pm 2 \sigma$ residual scatter in the relation (encompassing all of the standards). Stars above this line are clearly chromospherically active, however those within the $2 \sigma$ scatter have $\mathrm{H} \alpha$ emission similar to older field stars. 


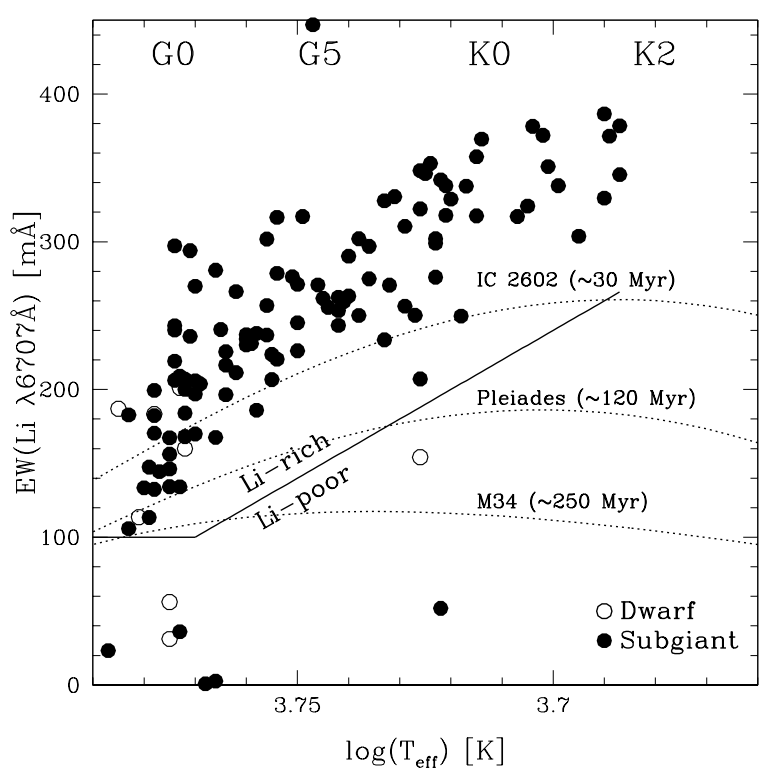

Fig. 3.- EWs for Li I $\lambda 6707$ for the program stars compared to regression fits for stars in young open clusters (see §4.2.2). We discuss assignment of dwarf and subgiant luminosity classes in $§ 4.1 .3$. The pre-MS candidates form an obvious locus, and we select all stars above the solid line as "Li-rich".

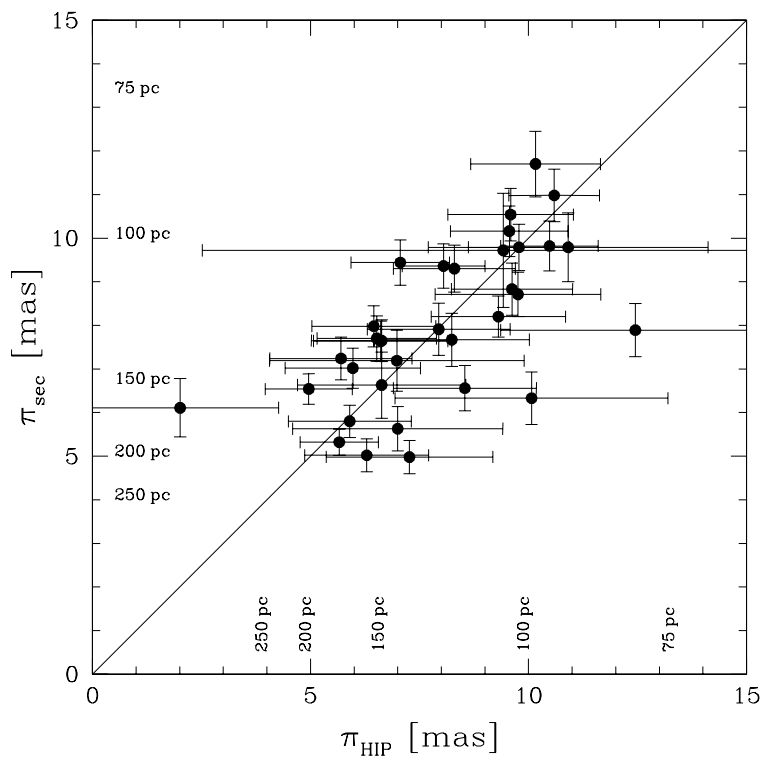

Fig. 4.- Comparison between Hipparcos astrometric parallaxes and our secular parallaxes calculated using the moving group method. Data points are Pre-MS (and Pre-MS?) association members from Tables 6 and 5 .

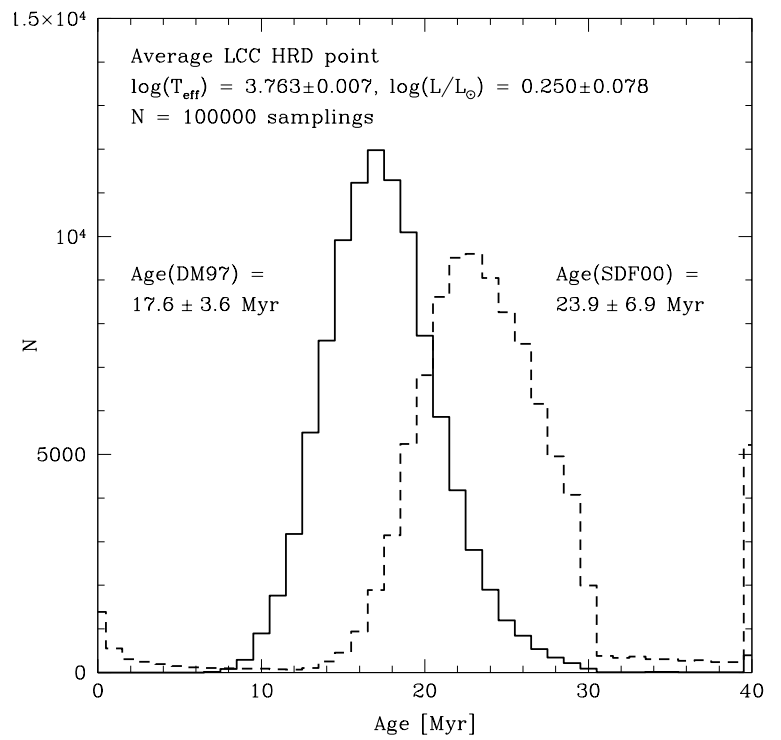

Fig. 5.- A histogram of the inferred ages from the DM97 and SDF00 tracks for a hypothetical LCC Pre-MS star with average H-R diagram point $\left(T_{e f f}, \log \mathrm{L} / \mathrm{L}_{\odot}\right)$ and gaussian uncertainties. The extreme right bin retains all points older than 40 Myr. The standard deviations are calculated using only stars with ages between 1-100 Myr. 


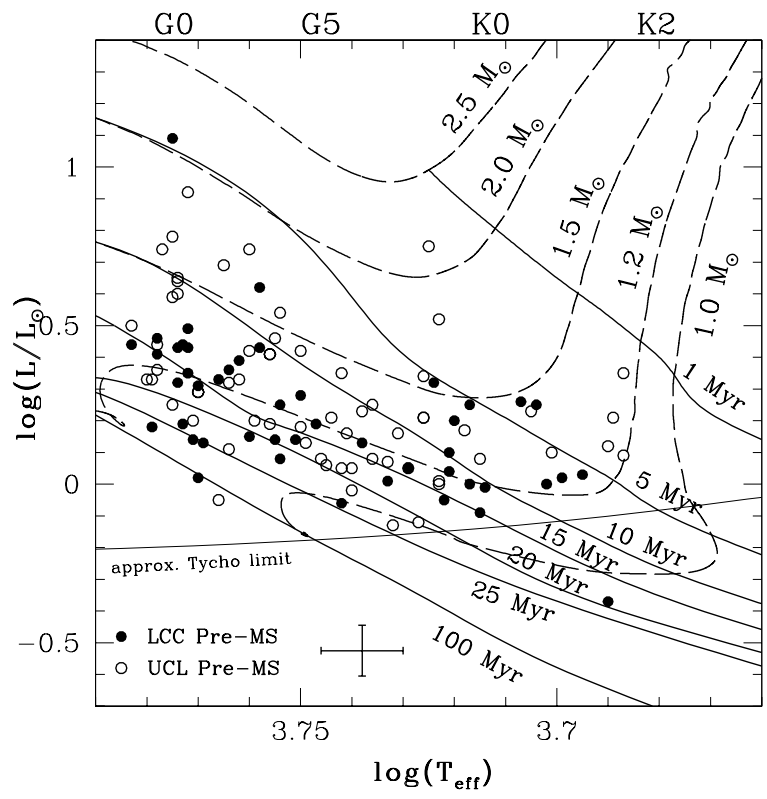

Fig. 6.- Theoretical H-R diagram for stars identified as Pre-MS or Pre-MS? in Tables 5 and 6 in the UCL (open circles) and LCC samples (filled circles). The pre-MS evolutionary tracks of DM97 are overlayed. The ACT/TRC magnitude limit (V $=11 \mathrm{mag})$ is shown for a distance of $150 \mathrm{pc}\left(\mathrm{A}_{V}\right.$ $=0.3$ assumed $)$. The star in the bottom right corner (TYC 8648-446-1) is one of the faintest stars in our sample $(\mathrm{V}=11.2 \mathrm{mag})$ with larger than average errors in $\log \mathrm{L} / \mathrm{L}_{\odot}$ - hence its unusual position. The average $1 \sigma$ error bars in $\log \mathrm{T}_{\text {eff }}$ and $\log \mathrm{L} / \mathrm{L}_{\odot}$ are shown.

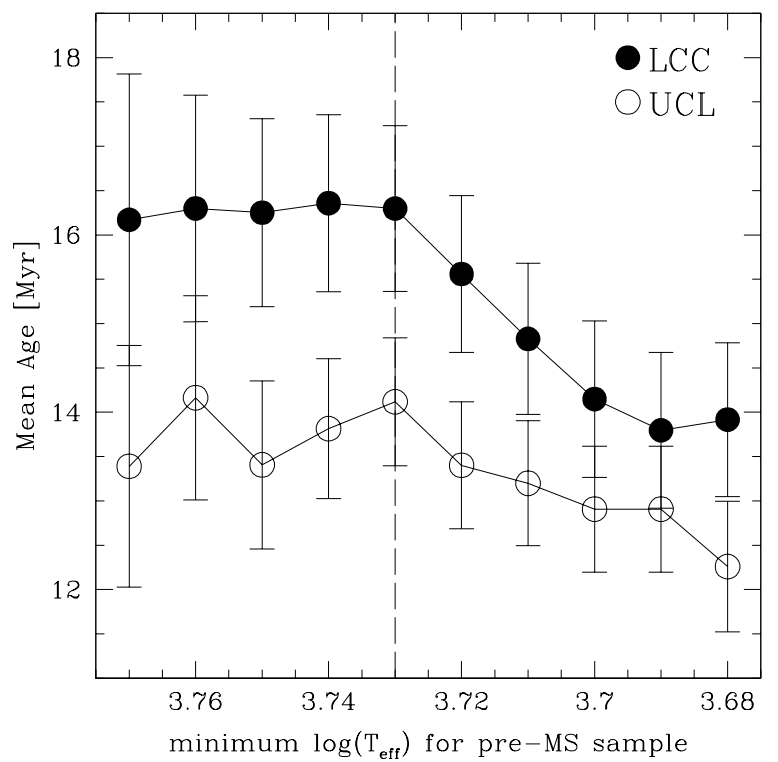

Fig. 7.- Illustration of the effects of magnitude bias on our mean age estimates for the preMS populations. The abscissa is the minimum $\log \left(T_{\text {eff }}\right)$ threshold for evaluating the mean sample ages (using DM97 tracks). The ordinate is calculated mean age with standard errors of the mean (shown; typically $\approx 1 \mathrm{Myr}$ ). At cooler temperatures (later than K0), the magnitude limit of our survey biases the sample towards more luminous stars, thereby decreasing the mean age estimate. From this diagram, we choose $\log \mathrm{T}_{\text {eff }}=3.73$ (vertical dashed line) as the lower $T_{\text {eff }}$ cut-off for evaluating the mean pre-MS ages. Known spectroscopic binaries are included here, but excluded in the final age estimates presented in Table 8 . The observed isochronal ages and spread are $16 \pm 5$ Myr for LCC and $14 \pm 5$ Myr for UCL. 

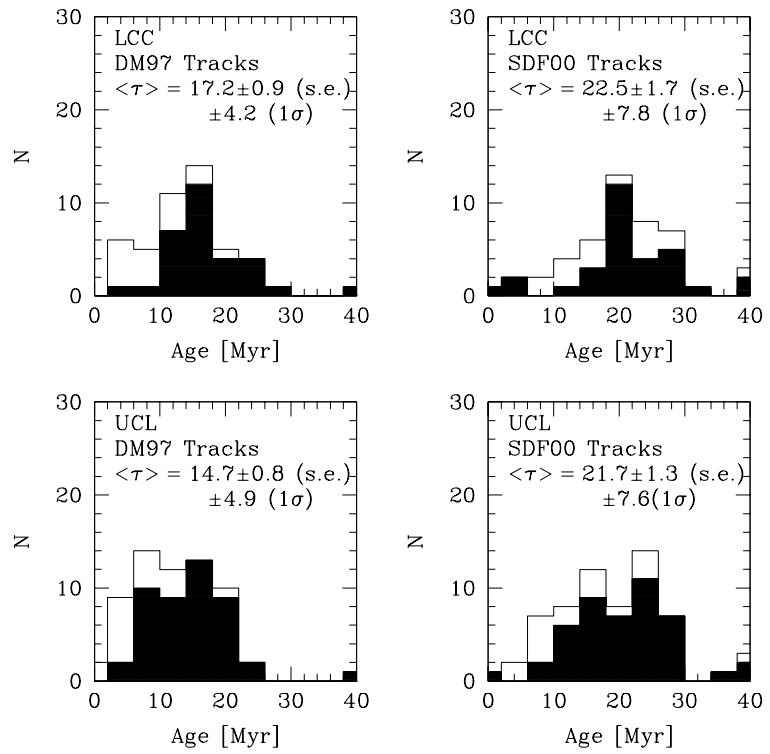

Fig. 8.- Histograms of the isochronal ages for pre-MS and "pre-MS?" candidates in Tables 5 and 6 from the models of DM97 and SDF00. The filled bins are for stars with $\log \mathrm{T}_{\text {eff }}>3.73$, and the unfilled bins are for the entire (magnitude-biased) sample. Mean isochronal ages (with standard errors of the means and $1 \sigma$ uncertainties) are given for the unbiased sample $\left(\log \mathrm{T}_{\text {eff }}>3.73\right)$. Outliers with isochronal ages of $>40 \mathrm{Myr}$ are counted within the $40 \mathrm{Myr}$ bin.

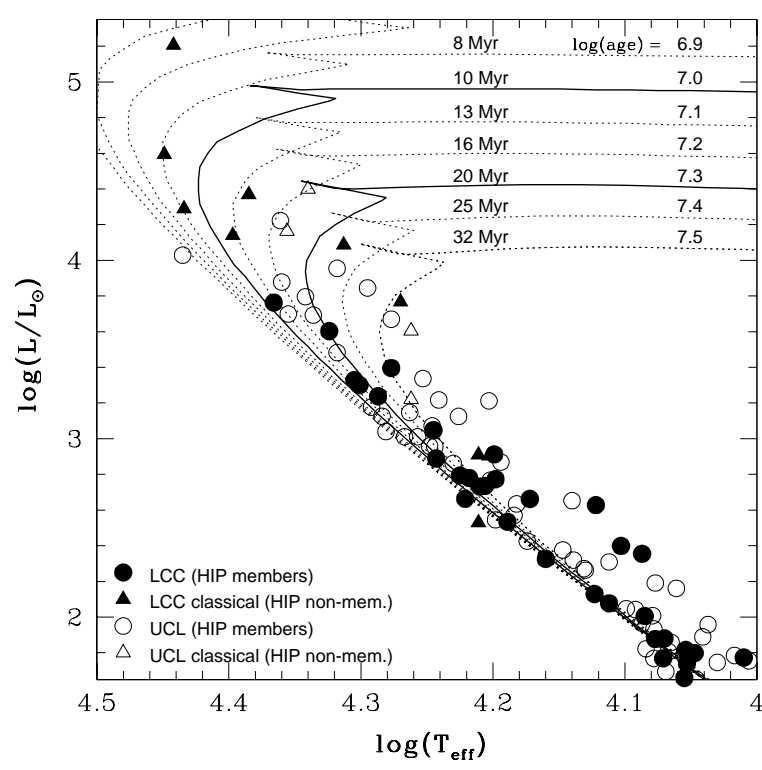

Fig. 9.- Theoretical H-R diagram for the Bstar candidate members of the LCC \& UCL memberships using the evolutionary tracks of Bertelli et al. (1994). Only the most massive Hipparcos members were included in the age estimates. The unusual variable HIP 67472 ( $\mu$ Cen; B2Vnpe; $\log \mathrm{L} / \mathrm{L}_{\odot}, \log \mathrm{T}_{\text {eff }}=4.43,4.0$ ) was excluded from the UCL turn-off age estimate. 


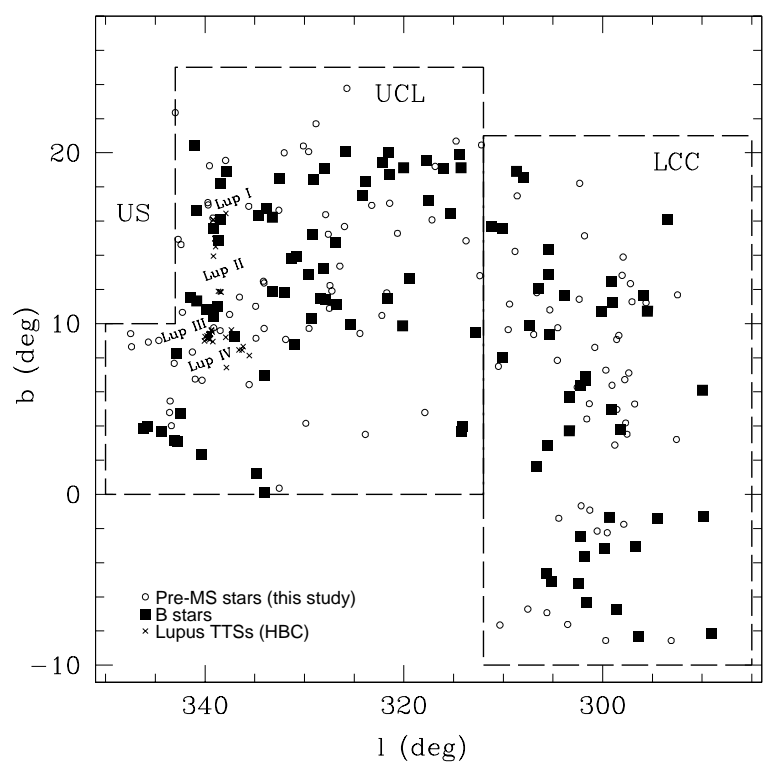

Fig. 10.- Map of the UCL and LCC subgroups of the Sco-Cen OB association (Sco OB2). The B-star population from de Zeeuw et al. (1999) is shown by filled squares. The pre-MS (and preMS?) sample from this survey is shown as open circles. Pre-ROSAT T Tauri stars in the HBC catalog associated with the Lupus cloud are shown as Xs (Herbig \& Bell 1988). 
TABLE 1

Properties of de Zeeuw et al. (1999) Sco-Cen G-K Candidate Members

\begin{tabular}{|c|c|c|c|c|c|c|c|c|}
\hline $\begin{array}{cc}(1) & (2) \\
\text { Name OB } & \text { OBP } \\
\text { HIP Grp. }\end{array}$ & 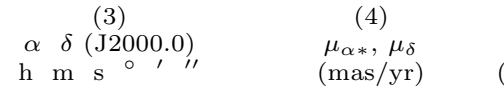 & $\begin{array}{c}(5) \\
\mathrm{V} \\
(\mathrm{mag})\end{array}$ & $\begin{array}{l}(6) \\
B-V \\
(\mathrm{mag})\end{array}$ & $\begin{array}{l}(7) \\
\text { SpT } \\
\text { MSS }\end{array}$ & $\begin{array}{c}(8) \\
\text { J } \\
(\mathrm{mag})\end{array}$ & $\begin{array}{c}(9) \\
\stackrel{H}{(m a g)}\end{array}$ & $\begin{array}{l}(10) \\
\mathrm{K}_{s} \\
(\mathrm{mag})\end{array}$ & $\begin{array}{l}(11) \\
\text { notes }\end{array}$ \\
\hline 57524 LCC 11 & $: 24.55-49: 53: 03.0-3$ & 9.07 & $0.63 \pm 0.02$ & $\mathrm{G} 3 / 5 \mathrm{VPq} 2$ & $7.91 \pm 0.02$ & $.59 \pm 0 .($ & $.51 \pm 0.0$ & 2 1RXS J114724.3-495250, TWA 19A \\
\hline LCC 12 & $: 05: 47.48-51: 00: 12.1-37.5 \pm 0.8-11.5 \pm 0.8$ & 8.89 & $0.63 \pm 0.02$ & G1Vq1 & $7.67 \pm 0.01$ & $7.37 \pm 0.03$ & $7.27 \pm 0.01$ & $1 \operatorname{var}(0.06), 1 \mathrm{RXS} J 120547.8-510007$ \\
\hline 59854 LCC 12 & $: 16: 27.84-50: 08: 35.8-29.1 \pm 1.3-8.6 \pm 1.0$ & 9.34 & $0.67 \pm 0.02$ & G3Vq1 & $8.04 \pm 0.01$ & $7.69 \pm 0.02$ & $7.61 \pm 0.03$ & 3 1RXS J121627.9-500829 \\
\hline 60885 LCC 12 & $: 28: 40.05-55: 27: 19.3-34.9 \pm 0.8-16.0 \pm 0.7$ & 8.89 & $0.64 \pm 0.02$ & G1q4 & $7.68 \pm 0.02$ & $7.40 \pm 0.05$ & $7.28 \pm 0.03$ & $3 \operatorname{var}(0.02), 1 \mathrm{RXS}$ J122840.3-552707 \\
\hline 60913 LCC 12 & $: 29: 02.25-64: 55: 00.6-37.5 \pm 1.2-11.4 \pm 0.9$ & 9.04 & $0.73 \pm 0.02$ & G5Vq1 & $7.60 \pm 0.01$ & $7.26 \pm 0.01$ & $7.14 \pm 0.01$ & \\
\hline 62445 LCC 12 & $: 47: 51.87-51: 26: 38.2-30.4 \pm 1.0 \quad-8.7 \pm 1.0$ & 9.52 & $0.80 \pm 0.03$ & G8/K0Vq3 & $7.79 \pm 0.01$ & $7.38 \pm 0.03$ & $7.25 \pm 0.01$ & $1 \operatorname{var}(0.10), 1 \mathrm{RXS}$ J124751.7-512638 \\
\hline 63797 LCC 13 & $: 04: 30.96-65: 55: 18.5-42.4 \pm 1.0-14.2 \pm 0.8$ & 8.48 & $0.74 \pm 0.01$ & G3Vq1 & $7.02 \pm 0.04$ & $6.79 \pm 0.02$ & $6.71 \pm 0.01$ & \\
\hline 63847 LCC 13 & $: 05: 05.29-64: 13: 55.3-36.5 \pm 1.1-19.5 \pm 1.3$ & 9.18 & $0.73 \pm 0.02$ & G5Vq4 & $7.78 \pm 0.01$ & $7.44 \pm 0.03$ & $7.36 \pm 0.03$ & $3 \operatorname{var}(0.03)$ \\
\hline 65423 LCC 13 & $: 24: 35.12-55: 57: 24.2-29.0 \pm 1.0-13.0 \pm 1.0$ & 9.59 & $0.66 \pm 0.03$ & $(\mathrm{G} 3 \mathrm{w}) \mathrm{F} 7 \mathrm{q} 2$ & $8.45 \pm 0.01$ & $8.16 \pm 0.03$ & $8.10 \pm 0.01$ & 1 1RXS J132435.3-555719 \\
\hline 65517 LCC 13 & $: 25: 47.83-48: 14: 57.9-39.0 \pm 1.2-20.3 \pm 1.0$ & 9.76 & $0.60 \pm 0.04 \mathrm{~K}$ & $0 / 2 \mathrm{~V}+(\mathrm{G}) \mathrm{q}^{3}$ & $8.50 \pm 0.02$ & $8.18 \pm 0.03$ & $8.08 \pm 0.03$ & $3 \operatorname{var}(0.05, P=1.09 \mathrm{~d}), \mathrm{V} 966 \mathrm{Cen}, 1$ \\
\hline 66001 LCC 13 & $: 31: 53.61-51: 13: 33.1-29.0 \pm 1.5-20.1 \pm 1.1$ & 9.84 & $0.72 \pm 0.04$ & $\mathrm{G} 5 / 6 \mathrm{Vq} 3$ & $8.43 \pm 0.01$ & $8.00 \pm 0.03$ & $7.83 \pm 0.01$ & 1 1RXS J133152.6-511335 \\
\hline 66941 LCC 13 & $: 43: 08.69-69: 07: 39.5-32.7 \pm 0.7-19.8 \pm 0.9$ & 7.57 & $0.74 \pm 0.01$ & G2IV/Vq2 & $6.21 \pm 0.01$ & $5.85 \pm 0.03$ & $5.77 \pm 0.01$ & $1 \operatorname{var}(0.08)$, CCDM $1343-6908,1 \mathrm{RXS} \mathrm{J} 1$ \\
\hline 67522 UCL 13 & $: 50: 06.28-40: 50: 08.8-29.3 \pm 1.5-22.9 \pm 1.0$ & 9.79 & $0.67 \pm 0.04$ & G1Vq2 & $8.58 \pm 0.01$ & $8.30 \pm 0.04$ & $8.16 \pm 0.02$ & 2 1RXS J135005.7-405001 \\
\hline 68726 UCL 14 & $: 04: 07.12-37: 15: 50.5-16.9 \pm 0.8-16.7 \pm 0.7$ & 7.11 & $0.72 \pm 0.02$ & $\mathrm{G} 3 \mathrm{IV} / \mathrm{Vq} 3$ & $5.29 \pm 0.00$ & $5.40 \pm 0.04$ & $4.92 \pm 0.05$ & 5 CCDM $14041-3716$ \\
\hline 71178 UCL 14 & $: 33: 25.78-34: 32: 37.7-28.1 \pm 1.6-28.0 \pm 1.4$ & 10.18 & $0.81 \pm 0.06$ & G8/K0Vq3 & $8.52 \pm 0.01$ & $8.06 \pm 0.02$ & $7.94 \pm 0.02$ & $2 \operatorname{var}(0.15)$, V1009 Cen \\
\hline 72070 UCL 14 & $: 44: 30.96-39: 59: 20.6-20.9 \pm 1.5-24.0 \pm 1.4$ & 9.32 & $0.64 \pm 0.03$ & G3Vq3 & $8.19 \pm 0.01$ & $7.93 \pm 0.02$ & $7.81 \pm 0.01$ & \\
\hline 74501 UCL 15 & $: 13: 29.22-55: 43: 54.6-16.1 \pm 0.8-24.0 \pm 0.8$ & 7.47 & $0.78 \pm 0.01$ & G2IVq1 & $5.86 \pm 0.01$ & $5.48 \pm 0.02$ & $5.20 \pm 0.01$ & \\
\hline 75924 UCL 15 & $: 30: 26.29-32: 18: 11.6-31.7 \pm 2.4-31.9 \pm 2.3$ & 8.80 & $0.65 \pm 0.03$ & G6Vq2 & $7.37 \pm 0.01$ & $7.05 \pm 0.03$ & $6.92 \pm 0.02$ & 2 1RXS J153026.1-321815 \\
\hline 76472 UCL 15 & $: 37: 04.66-40: 09: 22.1-20.2 \pm 1.7-27.6 \pm 1.4$ & 9.39 & $0.73 \pm 0.03$ & G5Vq2 & $7.98 \pm 0.01$ & $7.63 \pm 0.05$ & $7.52 \pm 0.01$ & 1 1RXS J153706.0-400929 \\
\hline 77015 UCL 15 & $: 43: 29.86-38: 57: 38.6-20.5 \pm 1.6-31.7 \pm 1.2$ & 9.66 & $0.61 \pm 0.03$ & G3Vq1 & $8.61 \pm 0.01$ & $8.34 \pm 0.03$ & $8.32 \pm 0.02$ & \\
\hline 77081 UCL 15 & $: 44: 21.05-33: 18: 55.0-19.1 \pm 1.8-29.5 \pm 1.4$ & 9.69 & $0.75 \pm 0.04$ & G8Vq2 & $8.27 \pm 0.02$ & $7.86 \pm 0.03$ & $7.79 \pm 0.02$ & \\
\hline 77135 UCL 15 & $: 44: 57.69-34: 11: 53.7-20.6 \pm 3.3-25.9 \pm 2.5$ & 9.88 & $0.78 \pm 0.02 \mathrm{GG}$ & $6 / \mathrm{G} 8 \mathrm{IV} / \mathrm{Vq} 3$ & $8.43 \pm 0.02$ & $8.04 \pm 0.02$ & $8.23 \pm 0.04$ & 4 CCDM $15450-3412$ \\
\hline 77144 UCL 15 & $: 45: 01.83-40: 50: 31.0-19.4 \pm 1.3-31.1 \pm 1.4$ & 9.46 & $0.57 \pm 0.03$ & G1Vq1 & $8.30 \pm 0.01$ & $7.97 \pm 0.03$ & $7.88 \pm 0.02$ & $2 \operatorname{var}(0.11), 1 \mathrm{RXS}$ J154502.0-4 \\
\hline 77524 UCL 15 & $: 49: 44.98-39: 25: 09.1-24.5 \pm 2.0-25.2 \pm 1.8$ & 10.64 & $1.09 \pm 0.12$ & $\mathrm{~K} 0(\mathrm{~V}) \mathrm{q} 3$ & $8.81 \pm 0.01$ & $8.27 \pm 0.02$ & $8.13 \pm 0.02$ & 2 1RXS J154944.7-392509 \\
\hline 77656 UCL 15 & $: 51: 13.73-42: 18: 51.3-18.0 \pm 1.2-30.0 \pm 1.0$ & 9.58 & $0.74 \pm 0.04$ & G8Vq3 & $8.15 \pm 0.03$ & $7.78 \pm 0.06$ & $7.67 \pm 0.02$ & 2 1RXS J155113.5-421858 \\
\hline 79610 UCL 16 & $: 14: 43.02-38: 38: 43.5-14.1 \pm 3.4-29.4 \pm 3.2$ & 9.24 & $0.52 \pm 0.03$ & $\mathrm{G} 1 / \mathrm{G} 2 \mathrm{Vq} 1$ & $8.07 \pm 0.01$ & $7.85 \pm 0.03$ & $7.98 \pm 0.03$ & 3 CCDM $16147-3839$ \\
\hline 80636 UCL 16 & $: 27: 52.34-35: 47: 00.4-13.1 \pm 2.1-25.5 \pm 1.2$ & 9.37 & $0.68 \pm 0.03$ & $\mathrm{G} 6 \mathrm{Vq} 2$ & $8.04 \pm 0.01$ & $7.71 \pm 0.01$ & $7.62 \pm 0.01$ & 1 1RXS J162752.8-354702 \\
\hline 81380 UCL 16 & $: 37: 12.87-39: 00: 38.1-14.4 \pm 2.1-21.5 \pm 1.6$ & 9.82 & $0.66 \pm 0.05$ & $\mathrm{G} 2 / 5 \mathrm{Vq} 3$ & $8.45 \pm 0.02$ & $8.09 \pm 0.04$ & $7.99 \pm 0.03$ & \\
\hline 81447 UCL 16 & $: 38: 05.53-34: 01: 10.6-11.3 \pm 1.5-23.9 \pm 1.0$ & 9.08 & $0.54 \pm 0.05$ & G1IV/Vq1 & $7.91 \pm$ & $7.66 \pm 0$ & $7.55 \pm 0.03$ & \\
\hline 81775 UCL 16 & $: 42: 10.36-31: 30: 15.0-14.1 \pm 1.5-18.3 \pm 1.3$ & 9.44 & $0.64 \pm 0.04$ & G5Vq2 & $8.36 \pm 0.02$ & $8.37 \pm 0.05$ & $8.08 \pm 0.04$ & \\
\hline
\end{tabular}

Note.- Columns: (1) Hipparcos ID, (2) OB subgroup region, (3) J2000.0 position, (4) proper motion components (mas yr $\mathrm{yr}^{-1}$; where $\mu_{\alpha *}=\mu_{\alpha} \cos \delta$ ), (5) $\mathrm{V}$ magnitude (Johnson), (6) $B-V$ color (Johnson), (7) Spectral types from the Michigan Spectral Survey (Vol. 1-3); "q1" indicates the flag "quality = 1" in the MSS catalog, where q=1,2 stars are judged to have reliable spectral types, (8-10) 2MASS JHK $s$ magnitudes, (11) Notes on variability, X-ray counterparts, and multiple star system name (CCDM) from Hipparcos. "Var. $(N . N N)$ " indicates that the H6 field in the Hipparcos catalog is identified as being variable in the broad $H_{p}$ pass-band. The magnitude scatter N. NN in $H_{p}$ is listed, and period $P$ if found. Near-IR photometry is from the preliminary 2MASS database. The name is given of RASS-BSC X-ray sources within 40" of Hipparcos 
TABLE 2

Properties of RASS-ACT/TRC CANDidates

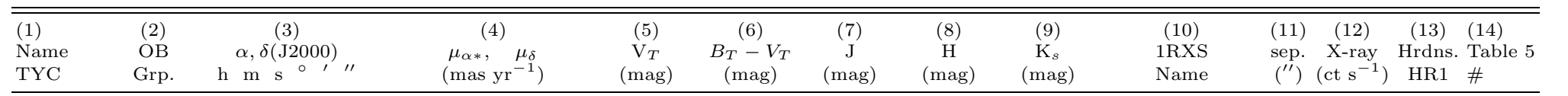

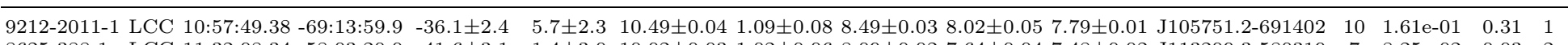

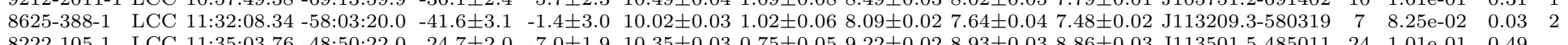

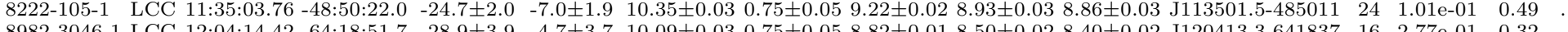

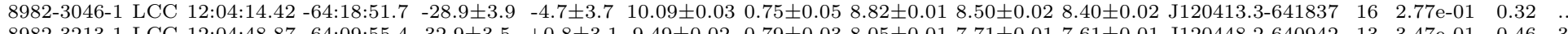

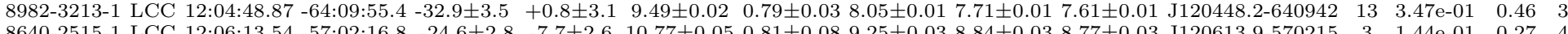

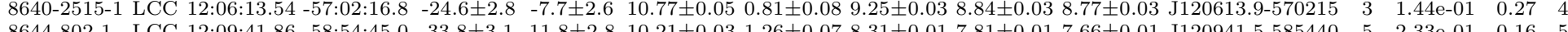

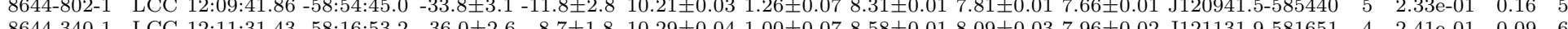

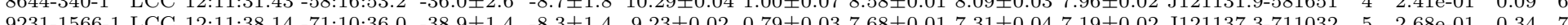

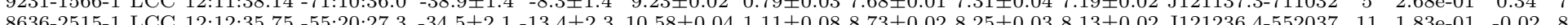

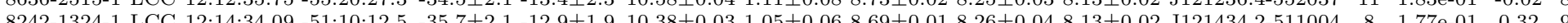

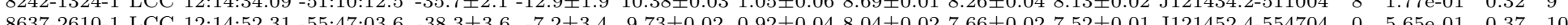
$\begin{array}{lllllllllll} & \end{array}$

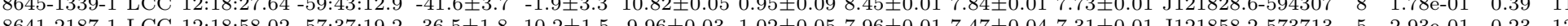

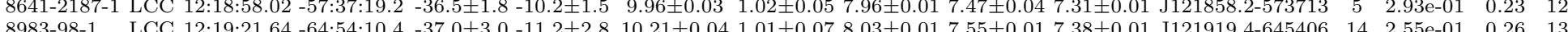

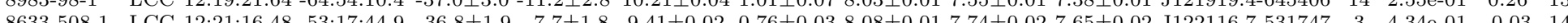

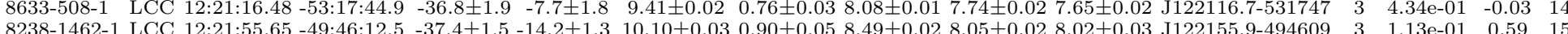

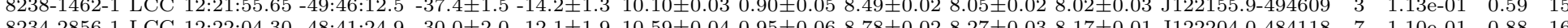

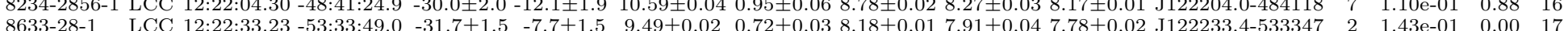

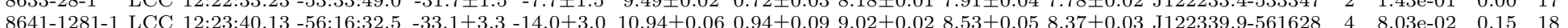

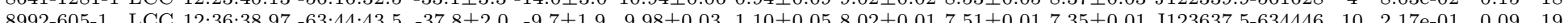
8646-166-1 LCC 12:36 $58.97-5412: 180-32.3 \pm 3.3-13.9 \pm 3.0 \quad 10.50 \pm 0.041 .07 \pm 0.078 .75 \pm 0.048 .28 \pm 0.038 .16 \pm 0.02 \quad J 123657.4541217 \quad 13 \quad 1.06-01 \quad 0.44 \quad 20$

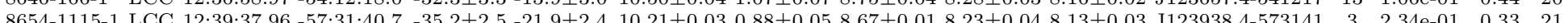

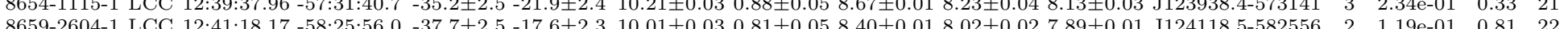

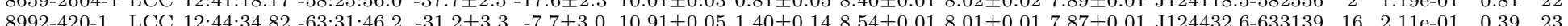

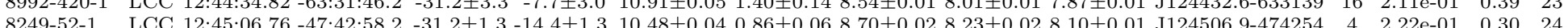

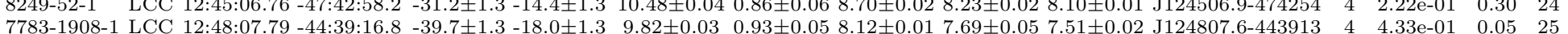

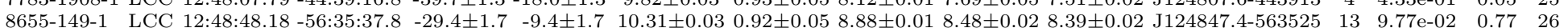

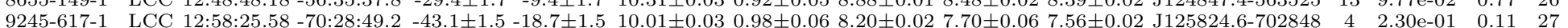

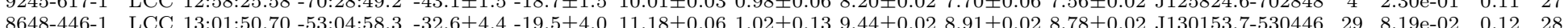

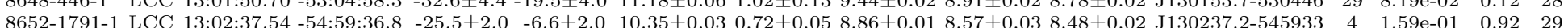

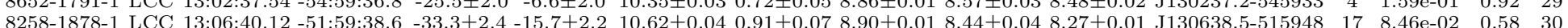

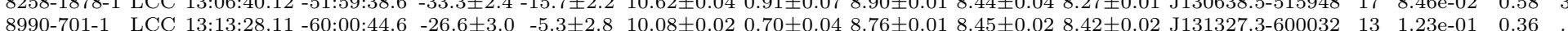

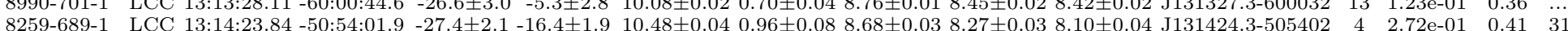

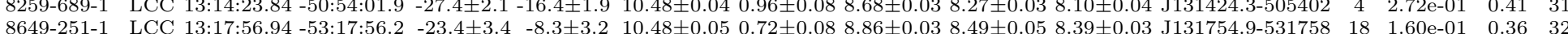

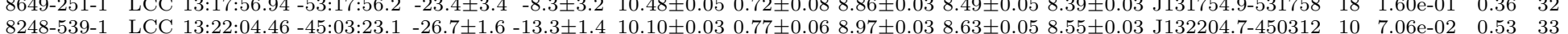

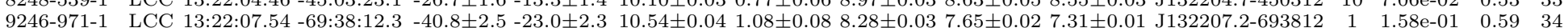

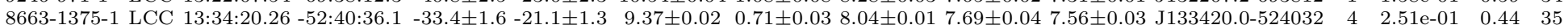

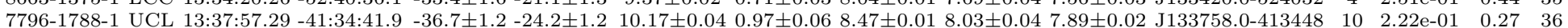

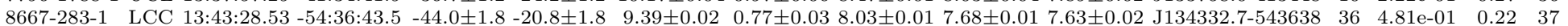

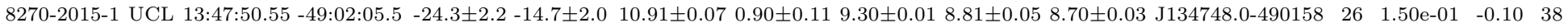

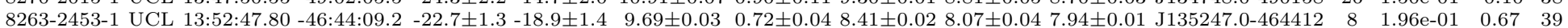

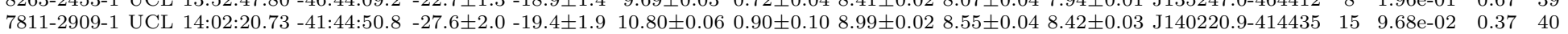

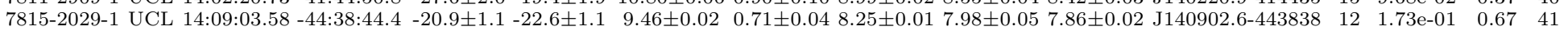

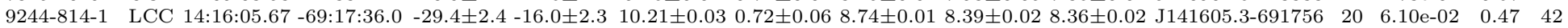

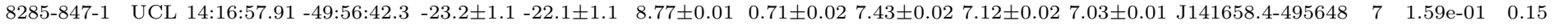

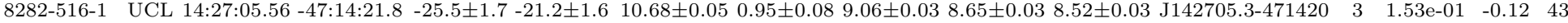


TABLE 2-Continued

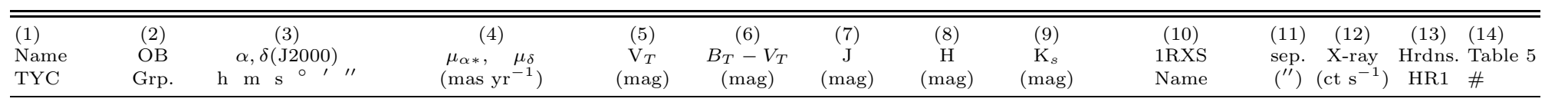

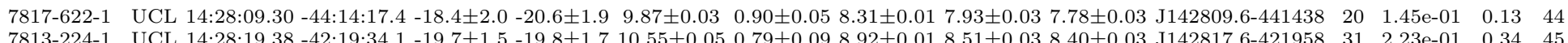
$\begin{array}{lllllllllllllll}7813-224-1 & \text { UCL } 14: 28: 19.38 & -42: 19: 34.1 & -19.7 \pm 1.5 & -19.8 \pm 1.7 & 10.55 \pm 0.05 & 0.79 \pm 0.09 & 8.92 \pm 0.01 & 8.51 \pm 0.03 & 8.40 \pm 0.03 & \mathrm{~J} 142817.6-421958 & 31 & 2.23 \mathrm{e}-01 & 0.34 & 45\end{array}$

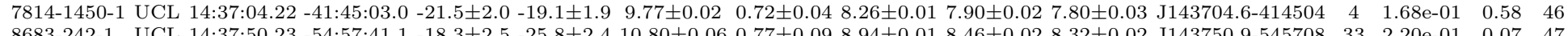

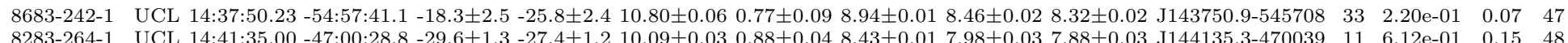

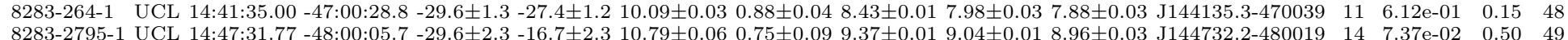

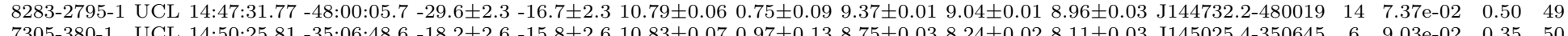

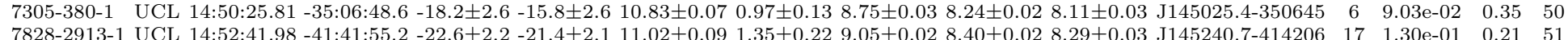

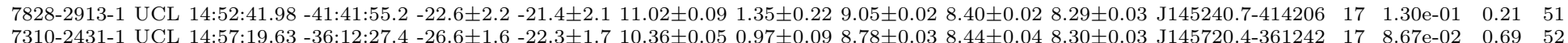

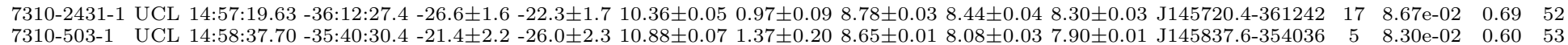

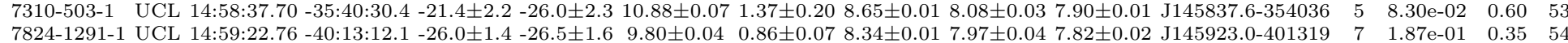

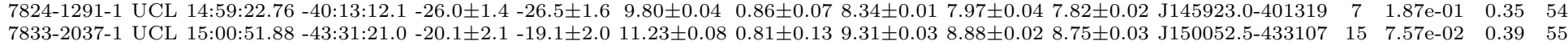

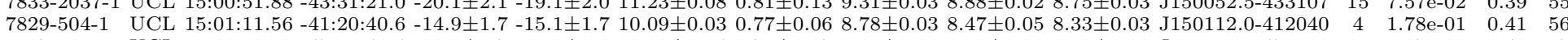

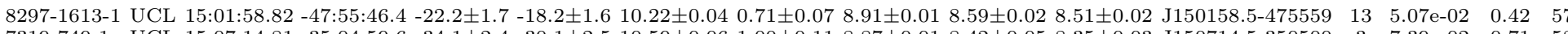

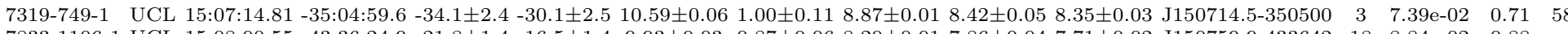

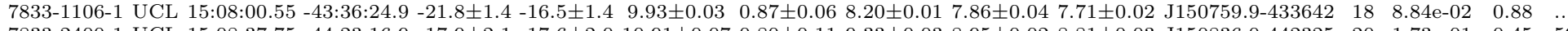

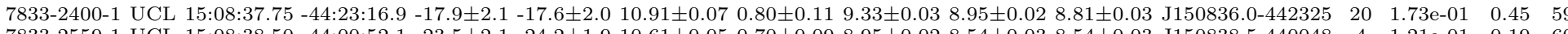

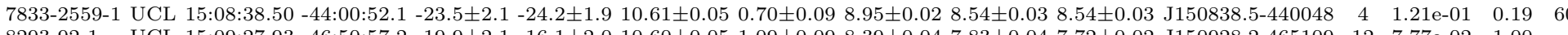
$\begin{array}{lllllllllllllll}8293-92-1 & \text { UCL } 15: 09: 27.93 & -46: 50: 57.2 & -19.9 \pm 2.1 & -16.1 \pm 2.0 & 10.60 \pm 0.05 & 1.09 \pm 0.09 & 8.39 \pm 0.04 & 7.83 \pm 0.04 & 7.72 \pm 0.02 & \mathrm{~J} 150928.2-465109 & 12 & 7.77 \mathrm{e}-02 & 1.00 & \ldots\end{array}$

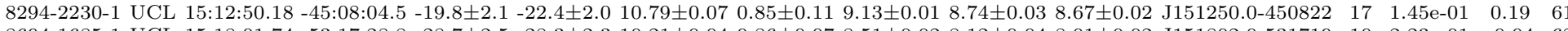

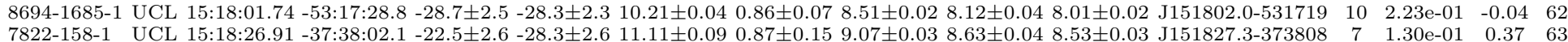

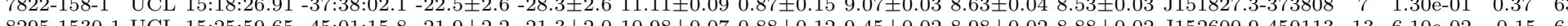

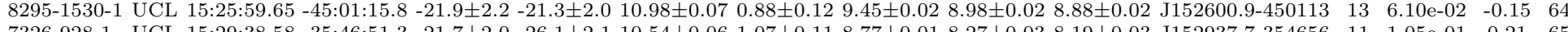

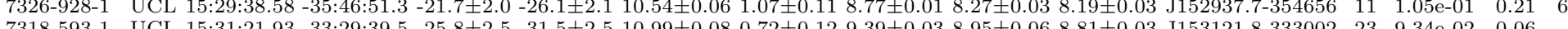

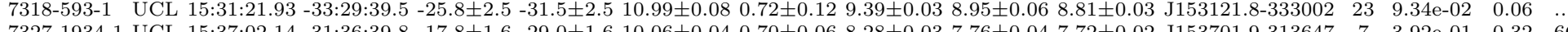

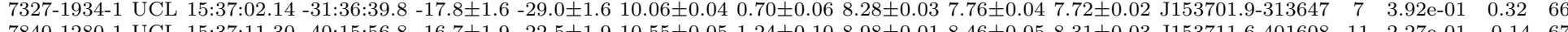

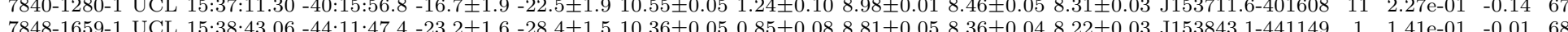

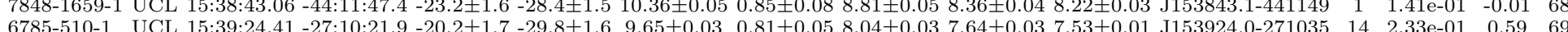
$\begin{array}{llllllllllll}6785-510-1 & & \end{array}$

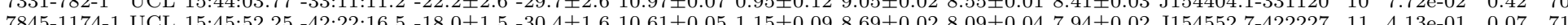

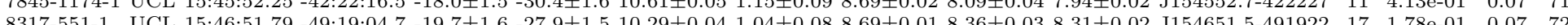

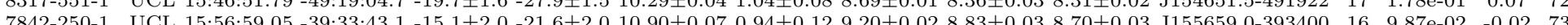
7333-1260-1

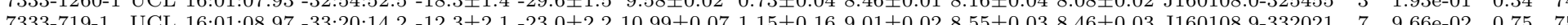

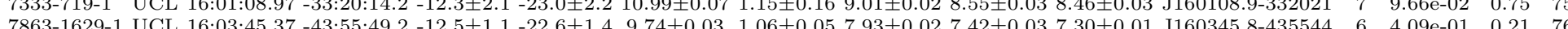

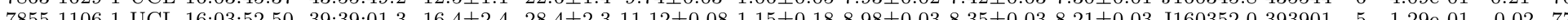

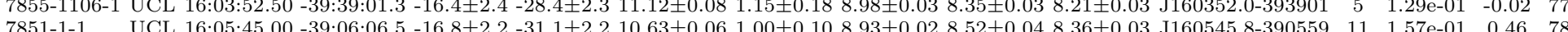

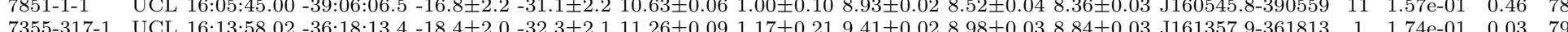

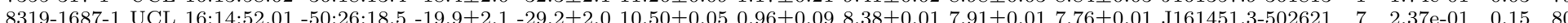

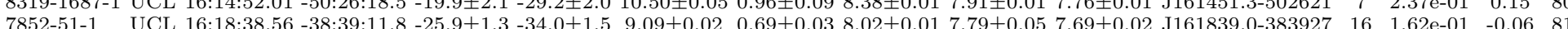

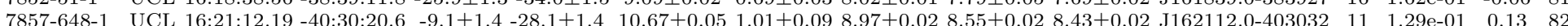

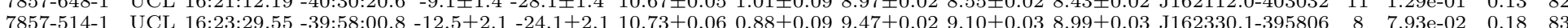

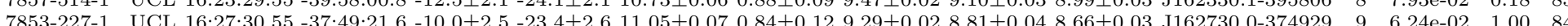

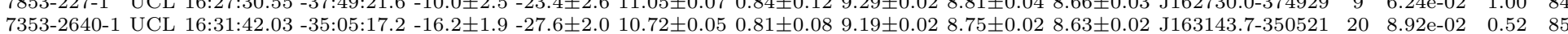

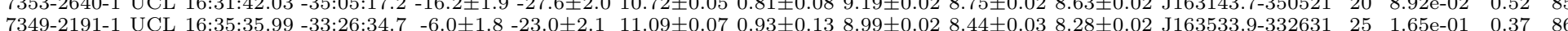

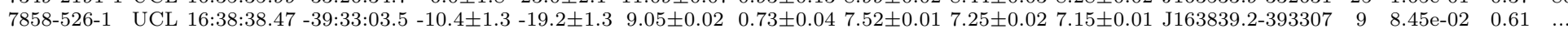


TABLE 2-Continued

\begin{tabular}{|c|c|c|c|c|c|c|c|c|c|c|c|c|c|}
\hline $\begin{array}{l}(1) \\
\text { Name } \\
\text { TYC }\end{array}$ & $\begin{array}{l}(2) \\
\text { OB } \\
\text { Grp. }\end{array}$ & $\begin{array}{c}(3) \\
\alpha, \delta(\mathrm{J} 2000) \\
\mathrm{h} \mathrm{m} \mathrm{s},{ }^{\circ}, "\end{array}$ & $\begin{array}{c}(4) \\
\mu_{\alpha *}, \quad \mu_{\delta} \\
\left(\operatorname{mas} \mathrm{yr}^{-1}\right)\end{array}$ & $\begin{array}{c}(5) \\
\mathrm{V}_{T} \\
(\mathrm{mag})\end{array}$ & $\begin{array}{c}(6) \\
B_{T}-V_{T} \\
(\mathrm{mag})\end{array}$ & $\begin{array}{c}(7) \\
\mathrm{J} \\
(\mathrm{mag})\end{array}$ & $\begin{array}{c}(8) \\
\stackrel{H}{(m a g)}\end{array}$ & $\begin{array}{c}(9) \\
\mathrm{K}_{s} \\
(\mathrm{mag})\end{array}$ & $\begin{array}{l}(10) \\
1 \mathrm{RXS} \\
\text { Name }\end{array}$ & $\begin{array}{l}\text { (11) } \\
\text { sep. } \\
\left({ }^{\prime \prime}\right)\end{array}$ & $\begin{array}{c}(12) \\
\text { X-ray } \\
\left(\mathrm{ct} \mathrm{s}^{-1}\right)\end{array}$ & $\begin{array}{c}(13) \\
\text { Hrdns. } \\
\text { HR1 }\end{array}$ & $\begin{array}{l}(14) \\
\text { Table } 5 \\
\#\end{array}$ \\
\hline $\begin{array}{l}7858-830-1 \\
7871-1282-1\end{array}$ & $\begin{array}{l}\text { UCL } \\
\text { UCL }\end{array}$ & $: \begin{array}{l}39: 59.30-39: 24: 59.2 \\
: 42: 24.00-40: 03: 29.7\end{array}$ & $\begin{array}{l}-9.7 \pm 2.2-18.9 \pm 2.2 \\
-11.1 \pm 1.5-20.2 \pm 1.3\end{array}$ & $\begin{array}{c}10.68 \pm 0.08 \\
9.70 \pm 0.03\end{array}$ & $\begin{array}{r}0.84 \pm 0.12 \\
0.88 \pm 0.06\end{array}$ & $\begin{array}{l}8.67 \pm 0.02 \\
8.03 \pm 0.03\end{array}$ & $\begin{array}{l}8.26 \pm 0.04 \\
7.63 \pm 0.05\end{array}$ & $\begin{array}{l}8.12 \pm 0.02 \\
7.46 \pm 0.02\end{array}$ & $\begin{array}{l}\text { J163958.7-392457 } \\
\text { J164224.5-400329 }\end{array}$ & $\begin{array}{l}7 \\
5\end{array}$ & $\begin{array}{l}7.39 \mathrm{e}-02 \\
9.83 \mathrm{e}-02\end{array}$ & $\begin{array}{l}0.96 \\
0.44\end{array}$ & $\begin{array}{l}87 \\
88\end{array}$ \\
\hline
\end{tabular}

Note.- Columns: (1) Tycho-2 name, (2) OB subgroup region, (3) J2000.0 position from Tycho-2 catalog, (4) proper motion ( $\alpha^{*}, \delta$ components) in mas yr ${ }^{-1}$ (where

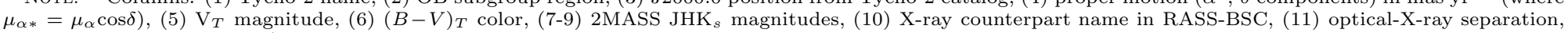
(12) X-ray count rate $\left(\mathrm{ct} \mathrm{s}^{-1}\right)$, (13) Hardness ratio HR1, (14) Number in Table 5 if star is found to be "pre-MS" or "pre-MS?" in nature. X-ray data is from ROSAT All-Sky Survey BSC (Voges et al. 1999), astrometry and optical photometry are from Tycho-2 catalog (Høg et al. 2000a), and near-IR photometry is from the 2MASS working database. 
TABLE 3

Spectral Standard Stars

\begin{tabular}{|c|c|c|c|c|c|c|c|c|c|}
\hline $\begin{array}{r}\text { Name } \\
\text { HD }\end{array}$ & $\begin{array}{c}\text { Name } \\
\text { HR }\end{array}$ & $\begin{array}{c}\text { MK } \\
\text { Sp.Type }\end{array}$ & $\begin{array}{c}\mathrm{V} \\
(\mathrm{mag})\end{array}$ & $\pi \underset{(\mathrm{mas})}{ \pm \sigma_{\pi}}$ & $\begin{array}{l}B-V \\
(\mathrm{mag})\end{array}$ & $\begin{array}{c}{[\mathrm{Fe} / \mathrm{H}]} \\
\text { adopted }\end{array}$ & $\begin{array}{c}\text { MSS } \\
\text { Sp.Type }\end{array}$ & $\begin{array}{l}\text { Adopted } \\
\text { Sp.Type }\end{array}$ & $\begin{array}{l}\text { notes } \\
\ldots\end{array}$ \\
\hline 182640 & 7377 & FoIV & 3.36 & $65.1 \pm 0.8$ & 0.32 & & $\mathrm{~F} 2 \mathrm{~V}$ & FoIV & $\mathrm{a}, \mathrm{b}$ \\
\hline 173677 & 7061 & F6V & 4.19 & $52.4 \pm 0.7$ & 0.48 & -0.1 & & F6IV-V & c \\
\hline 84117 & 3862 & F9V & 4.93 & $67.2 \pm 0.7$ & 0.53 & $\ldots$ & G0V & F9V & \\
\hline 121370 & 5235 & G0IV & 2.68 & $88.2 \pm 0.8$ & 0.58 & 0.2 & $\ldots$ & G0IV & b \\
\hline 89010 & 4030 & G1.5IV-V & 5.95 & $32.9 \pm 0.9$ & 0.66 & 0.0 & $\ldots$ & $\mathrm{G} 1.5 \mathrm{IV}-\mathrm{V}$ & \\
\hline 126868 & 5409 & G2IV & 4.84 & $24.2 \pm 1.0$ & 0.69 & 0.0 & G3V & G2III-IV & $\mathrm{a}, d$ \\
\hline 161239 & 6608 & G2IIIb & 5.73 & $26.1 \pm 0.6$ & 0.68 & & & G2IV & \\
\hline 146233 & 6060 & $\mathrm{G} 2 \mathrm{Va}$ & 5.49 & $71.3 \pm 0.9$ & 0.65 & 0.0 & G5V & $\mathrm{G} 2 \mathrm{~V}$ & $\mathrm{a}$ \\
\hline 94481 & 4255 & G4III & 5.65 & $8.0 \pm 0.8$ & 0.83 & & KoIII+(G) & G4III & \\
\hline 117176 & 5072 & $\mathrm{G} 4 \mathrm{~V}$ & 4.97 & $55.2 \pm 0.7$ & 0.71 & -0.1 & & G4IV-V & e \\
\hline 188376 & 7597 & G5IV & 4.70 & $42.0 \pm 0.9$ & 0.75 & -0.1 & G3/5III & G5IV & f \\
\hline 115617 & 5019 & G6.5V & 4.74 & $117.3 \pm 0.7$ & 0.71 & 0.0 & G5V & G6.5V & \\
\hline 114946 & 4995 & G7IV-V & 5.31 & $25.9 \pm 0.7$ & 0.86 & $-0.1:$ & G8III/IV & G7IV & \\
\hline 188512 & 7602 & G8IV & 3.71 & $73.0 \pm 0.8$ & 0.86 & $0.0:$ & $\ldots$ & G8IV & $\mathrm{a}$ \\
\hline 165760 & 6770 & G8III & 4.64 & $13.7 \pm 0.8$ & 0.95 & -0.1 & & G8III & \\
\hline 95272 & 4287 & $\mathrm{~K} 0+\mathrm{III}$ & 4.08 & $18.7 \pm 1.0$ & 1.08 & -0.1 : & K1III & $\mathrm{K} 0+\mathrm{III}$ & \\
\hline 131511 & 5553 & $\mathrm{~K} 0.5 \mathrm{~V}$ & 6.00 & $86.7 \pm 0.8$ & 0.84 & & & $\mathrm{~K} 0.5 \mathrm{~V}$ & $\mathrm{a}$ \\
\hline 165438 & 6756 & K1IV & 5.74 & $28.6 \pm 0.8$ & 0.97 & 0.0 & KOIV & K1IV & \\
\hline 131977 & 5568 & $\mathrm{~K} 4 \mathrm{~V}$ & 5.72 & $169.3 \pm 1.7$ & 1.02 & 0.0 & $\mathrm{~K} 4 \mathrm{~V}$ & $\mathrm{~K} 4 \mathrm{~V}$ & \\
\hline 120467 & $\ldots$ & K6Va & 8.16 & $70.5 \pm 1.0$ & 1.26 & $\ldots$ & $\ldots$ & $\mathrm{K} 6 \mathrm{~V}$ & \\
\hline
\end{tabular}

Note.- SB1,2 = spectroscopic binary in either SIMBAD or Duquennoy \& Mayor (1991). MSS = Michigan Spectral Survey Vols. 1-5 = Houk \& Cowley (1975), Houk (1978), Houk (1982), Houk \& Smith-Moore (1988), Houk \& Swift (1999). The $[\mathrm{Fe} / \mathrm{H}]$ estimate is adopted from the compilation of published values in Cayrel de Strobel et al. (2001). A semi-colon after the $[\mathrm{Fe} / \mathrm{H}]$ value indicates considerable scatter $(>0.2 \mathrm{dex})$ in the published estimates.

${ }^{a}$ SIMBAD lists as variable or suspected var., however Hipparcos finds scatter in $\mathrm{Hp} \leq 0.015$ mag. The typical scatter for the other standard stars was 0.005 mag in $\mathrm{Hp}$ (Hipparcos magnitude), with none greater than $0.01 \mathrm{mag}$.

${ }^{\mathrm{b}}$ Spectroscopic binary

${ }^{\mathrm{c}}$ From standard list of Garcia (1989), and originally in Johnson \& Morgan (1953), but not listed in either Keenan \& Yorka (1988) or Keenan \& McNeil (1989)

${ }^{\mathrm{d}}$ HR 5409 is a resolved binary (sep. 5") listed by SIMBAD as a variable star, however Hipparcos found the scatter in the Hp band to be only $0.015 \mathrm{mag}$.

${ }^{\mathrm{e}} \mathrm{HR} 5072$ is the planet host 70 Vir. Keenan \& McNeil (1989) call it G4V, however it is G5V in virtually every other reference (e.g. Gray, Graham, \& Hoyt (2001)). We retain Keenan's classification.

${ }^{\mathrm{f}}$ Hipparcos "G" binary 
TABLE 4

Stellar Classification Scheme

\begin{tabular}{lccccl}
\hline \hline \multirow{2}{*}{ Li- } & H $\alpha$ & \multicolumn{3}{l}{ Lum. N(R-T) N(HIP) Adopted } \\
Rich & Excess & Class & $\#$ & $\#$ & Class. \\
\hline Yes & Yes & IV & 94 & 7 & Pre-main sequence (PMS) \\
Yes & No & IV & 3 & 6 & Probably pre-main sequence? (PMS?) \\
Yes & Strong & IV & 1 & 0 & Pre-MS Classical T Tauri star (CTTS) \\
Yes & Yes/No & V & 4 & 1 & Young Dwarf (ZAMS) \\
No & Yes & V & 1 & 0 & Active Dwarf \\
No & Yes & IV & 4 & 0 & Active Subgiant \\
No & No & IV & 1 & 2 & Subgiant \\
No & No & III & 0 & 2 & Giant \\
No & No & V & 0 & 2 & Dwarf (MS) \\
No No(Wide) & IV & 2 & 0 & Chromosph.-Active Binary (CAB $)^{\mathrm{b}}$ \\
\hline$\cdots$ & $\cdots$ & $\cdots$ & 107 & 20 & Total \\
\hline
\end{tabular}

Note. - N $(\mathrm{R}-\mathrm{T})$ is number in RASS-ACT/TRC sample. N(HIP) is number in Hipparcos sample. "Li-rich" implies significant $\mathrm{Li}$ absorption, and above the line in Fig.3. "Active" means that the $\mathrm{H} \alpha$ equivalent widths are $>2 \times$ the $\sigma$-residual above the regression of values for field, standard stars (Fig. 2; Appendix C), implying that chromospheric emission is filling in the absorption line. Luminosity classes are assigned according to a star's placement in Fig. 1.

aincluded in Pre-MS sample count.

${ }^{\mathrm{b}}$ included in subgiant sample count. 
TABLE 5

LCC \& UCl Pre-Main Sequence Members from de Zeeuw et al. (1999) List

\begin{tabular}{|c|c|c|c|c|c|c|c|c|c|c|c|c|c|c|c|}
\hline $\begin{array}{l}\text { Name } \\
\text { HIP \# }\end{array}$ & $\begin{array}{l}\text { OB } \\
\text { Grp }\end{array}$ & $\begin{array}{l}\alpha, \delta(3) \\
\text { h m s d }{ }^{\prime \prime \prime}\end{array}$ & $\begin{array}{l}\pi_{H I P}^{(4)} \\
\text { (mas) }\end{array}$ & $\begin{array}{l}(5) \\
\pi_{s e c} \\
(\mathrm{mas})\end{array}$ & $\begin{array}{l}(6) \\
P_{1} \\
\%\end{array}$ & $\begin{array}{l}\overline{7(7)} \\
\mathrm{P}_{3} \\
\%\end{array}$ & $\begin{array}{l}\text { Spec. } \\
\text { Type }\end{array}$ & $\begin{array}{c}\log \left(T_{e f f}\right) \\
(\mathrm{K})\end{array}$ & $\begin{array}{c}\log \mathrm{L} / \mathrm{L} \\
(\mathrm{dex})\end{array}$ & $\begin{array}{l}(111) \\
A_{V} \\
(\mathrm{mag})\end{array}$ & 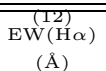 & $\begin{array}{c}(13) \\
\mathrm{EW}(\mathrm{Li}) \\
(\AA)\end{array}$ & $\begin{array}{c}(14) \\
\log \left(\mathrm{L}_{X}\right) \\
\left(\operatorname{erg~s}^{-1}\right)\end{array}$ & $\begin{array}{c}(15) \\
\log \left(\mathrm{L}_{X} /\right. \\
\left.\mathrm{L}_{b o l}\right)\end{array}$ & $\begin{array}{r}\text { DM97 } \\
\text { age,mass }\end{array}$ \\
\hline $\begin{array}{l}57524 \\
58996 \\
59854 \\
60885 \\
62913 \\
63845 \\
65517 \\
65517 \\
66001 \\
66941 \\
71172 \\
75924 \\
75472 \\
76472 \\
77081 \\
77135 \\
77144 \\
77524 \\
77656 \\
81386 \\
81480\end{array}$ & $\begin{array}{l}\text { LCC } \\
\text { LCC } \\
\text { LCC } \\
\text { LCC } \\
\text { LCC } \\
L C C \\
\text { LCC } \\
\text { LCC } \\
\text { LCC } \\
\text { UCL } \\
\text { UCL } \\
\text { UCL } \\
\text { UCL } \\
\text { UCL } \\
\text { UCL } \\
\text { UCL } \\
\text { UCL } \\
\text { UCL } \\
\text { UCL }\end{array}$ & 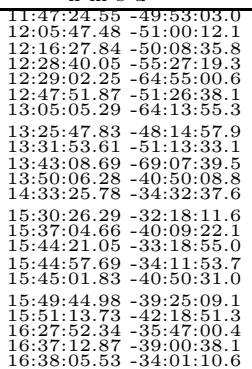 & 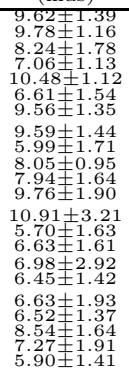 & 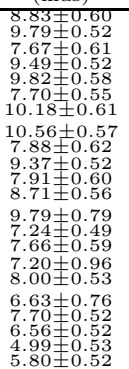 & $\begin{array}{l}1 \\
91 \\
99 \\
14 \\
100 \\
13 \\
48 \\
50 \\
82 \\
97 \\
93 \\
99 \\
16 \\
95 \\
69 \\
91 \\
43 \\
96 \\
100 \\
33 \\
92 \\
87\end{array}$ & $\begin{array}{c}97 \\
99 \\
100 \\
78 \\
100 \\
75 \\
89 \\
91 \\
97 \\
100 \\
99 \\
100 \\
57 \\
99 \\
92 \\
96 \\
86 \\
99 \\
100 \\
82 \\
99 \\
97\end{array}$ & 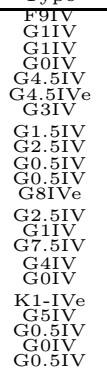 & 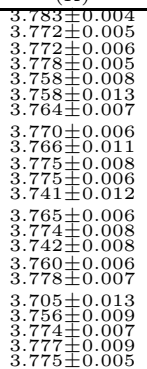 & 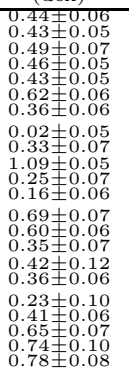 & 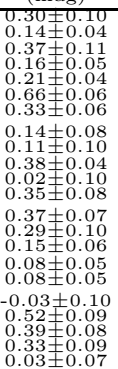 & $\begin{array}{l}.0 \\
3.3 \\
1.6 \\
2.7 \\
2.0 \\
-1.8 \\
1.6 \\
1.9 \\
1.2 \\
2.2 \\
2.2 \\
-0.2 \\
1.3 \\
0.4 \\
1.8 \\
2.2 \\
1.8 \\
-0.2 \\
1.7 \\
12.5 \\
2.5\end{array}$ & $\begin{array}{l}0.18 \\
0.21 \\
0.20 \\
0.13 \\
0.19 \\
0.24 \\
0.22 \\
0.17 \\
0.28 \\
0.16 \\
0.15 \\
0.26 \\
0.24 \\
0.24 \\
0.25 \\
0.23 \\
0.17 \\
0.32 \\
0.30 \\
0.21 \\
0.14 \\
0.13\end{array}$ & $\begin{array}{l}30.8 \\
30.5 \\
30.9 \\
30.9 \\
329.7 \\
30.7 \\
<29.7 \\
30.4 \\
30.4 \\
30.6 \\
31.1 \\
30.4 \\
<29.8 \\
30.9 \\
30.9 \\
30.8 \\
30.9 \\
30.5 \\
30.6 \\
30.5 \\
30.3 \\
30.9 \\
<30.3 \\
<30.2\end{array}$ & $\begin{array}{l}-3.3 \\
-3.6 \\
-3.2 \\
-3.8 \\
<-4.4 \\
<-3.4 \\
<-4.3 \\
-3.3 \\
-3.2 \\
-3.5 \\
-3.5 \\
<-3.9 \\
-2.9 \\
-3.9 \\
<-4.1 \\
-3.4 \\
-3.4 \\
-3.3 \\
-3.8 \\
-3.3 \\
<-4.0 \\
<-4.3\end{array}$ & 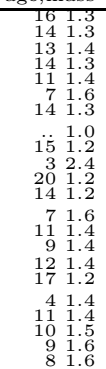 \\
\hline
\end{tabular}

Note.-Columns: (1) Hipparcos catalog ID, (2) OB subgroup, (3) J2000.0 position, (4) Hipparcos astrometric parallax (mas), (5) Secular parallax estimate (mas) (see §6.3), (6) Membership probability (with $v_{d i s p}=$ $\mathrm{km} \mathrm{s}^{-1}$ ), (8) Spectral type (see §4.1), (9) Effective temperature (see §6.2), (10) Luminosity (see §6.4), (11) Extinction A $V$ (see §6.4), (12) Equivalent Width of H $\alpha \lambda 6562.8(\AA)$ ), (13) Corrected EW of Li I $\lambda 6707.8(\AA$ upper limits assume RASS PSPC detection limit of $0.05 \mathrm{ct} \mathrm{s}^{-1}$ and $\left.\mathrm{HR} 1=0\right)$, (15) Logarithm of ratio between X-ray and bolometric luminosities, (16) Age (in Myr) and Mass (in

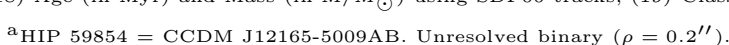

b ${ }^{2}$ IP 65517. The Michigan Spectral Survey (Houk 1978) classifies this star as K0/2(V) $+(\mathrm{G})$ (quality $=3$ ). We found it to be much earlier (G1.5), and note that the published $b-y$ and $B-V$ colors support an ${ }^{c}$ HIP $66941=$ HD $119022=$ Star "E" of Soderblom et al.'s (1998) high resolution survey of active southern stars. It is the most Li-rich of the very active stars in the Soderblom sample. We did not resolve this tight, e $\mathrm{d}_{\text {HIP }} 75924=$ CCDM J15304-3218AB. Both stars were on the slit $\left(\rho=1.5^{\prime \prime}\right)$.

$\mathrm{e}_{\text {HIP }} 77135=[$ KWS97 $]$ Lupus $126=$ CCDM J15450-3412AB. Binary is resolved $\left(\rho=3.4^{\prime \prime}\right)$ but both were on-slit.

$\mathrm{f}_{\text {HIP }} 77524=[\mathrm{KWS} 97]$ TTS $79=$ CCDM J15450-3412AB. Unresolved binary $\left(\rho=0.2^{\prime \prime}\right)$. 
TABLE 6

Pre-Main Sequence LCC \& UCL RASS-ACT/TRC Members

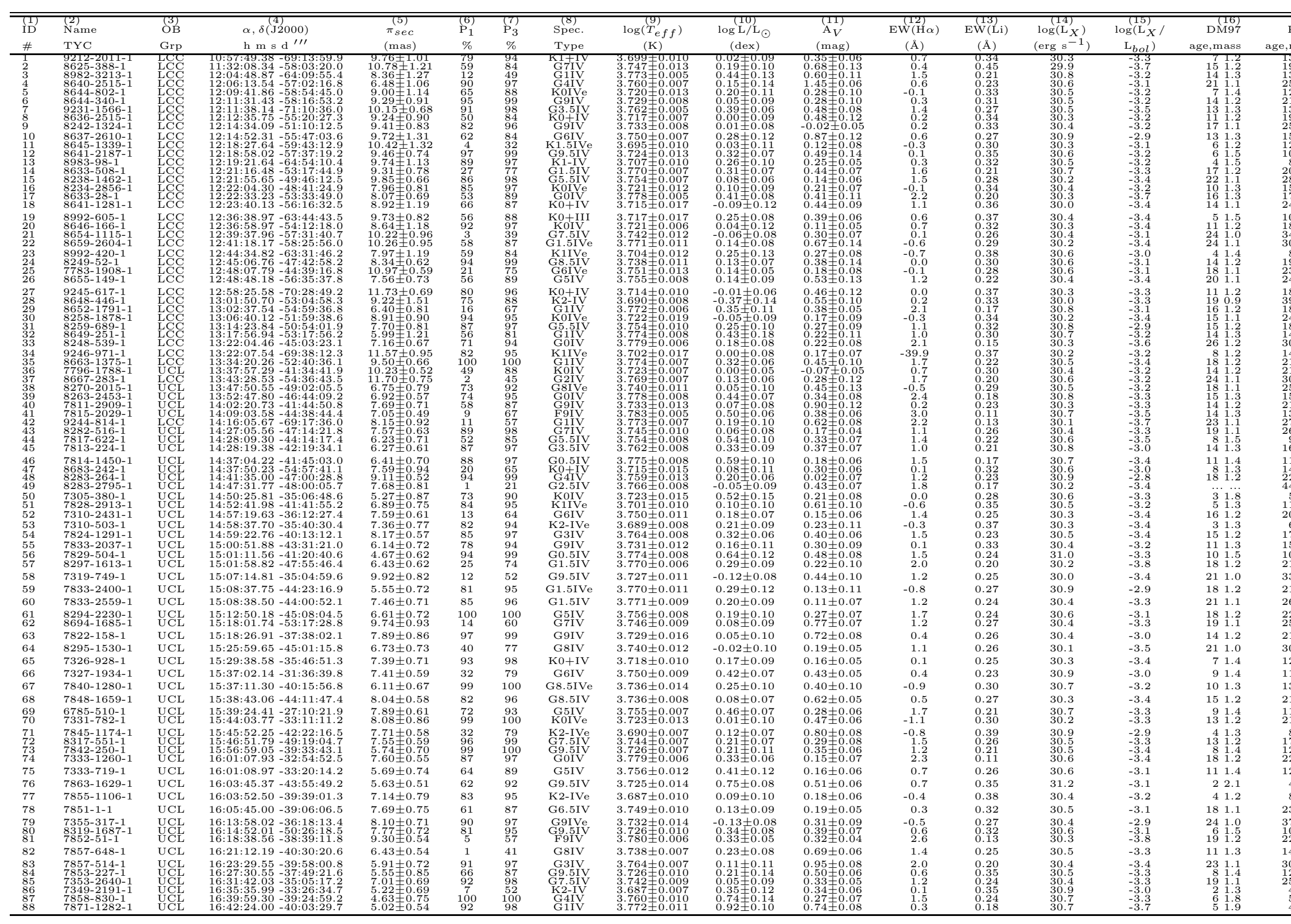

NoTE.-Columns: (1) short ID, (2) Tycho-2 name, (3) OB subgroup, (4) J200o.0 position, (5) Secular parallax estimate (mas) (see §̧6.3), (6) Membership probability (with $v_{d i s p}=1 \mathrm{~km} \mathrm{~s}^{-1}$ ), (7) Membership proba

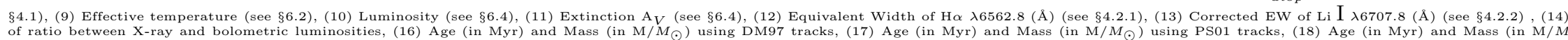


TABLE 7

RASS-ACT/TRC \& Hipparcos Stars Rejected as Sco-Cen Members

\begin{tabular}{|c|c|c|c|c|c|c|c|c|}
\hline $\begin{array}{l}(1) \\
\text { Name }\end{array}$ & $\begin{array}{c}(2) \\
\alpha, \delta(\mathrm{J} 2000) \\
\mathrm{h} \mathrm{m} \mathrm{s} \mathrm{d}^{\prime \prime \prime}\end{array}$ & $\begin{array}{l}(3) \\
\mathrm{P}_{1} \\
\% \\
\end{array}$ & $\begin{array}{l}(4) \\
\mathrm{P}_{3} \\
\% \\
\end{array}$ & $\begin{array}{c}(5) \\
\mathrm{SpT}\end{array}$ & $\begin{array}{c}(6) \\
\operatorname{EW}(\mathrm{H} \alpha) \\
(\AA)\end{array}$ & $\begin{array}{c}(7) \\
\operatorname{EW}(\mathrm{Li}) \\
(\AA)\end{array}$ & $\begin{array}{c}(8) \\
\log \left(\mathrm{L}_{X} /\right. \\
\left.\mathrm{L}_{b o l}\right) \\
\end{array}$ & $\begin{array}{l}(9) \\
\text { Type } \\
\ldots \\
\end{array}$ \\
\hline TYC 8222-105-1 & $11: 35: 03.76-48: 50: 22.0$ & 71 & 93 & $\mathrm{~F} 8.5 \mathrm{~V}$ & 2.7 & 0.19 & -3.4 & ZAMS \\
\hline TYC 8982-3046-1 & $12: 04: 14.42-64: 18: 51.7$ & 97 & 99 & G1V & 2.0 & 0.20 & -3.1 & ZAMS \\
\hline TYC 8990-701-1 & 13:13:28.11 -60:00:44.6 & 9 & 48 & F9V & 2.2 & 0.11 & -3.4 & ZAMS \\
\hline TYC $8285-847-1$ & $14: 16: 57.91-49: 56: 42.3$ & 43 & 87 & G2.5IV & 2.2 & 0.00 & -3.9 & subgiant $(\mathrm{CAB})$ \\
\hline TYC 7833-1106-1 & $15: 08: 00.55-43: 36: 24.9$ & 4 & 53 & G2IV & 0.6 & 0.00 & -3.5 & active subgiant \\
\hline TYC 8293-92-1 & $15: 09: 27.93-46: 50: 57.2$ & 28 & 71 & KOIV & 0.7 & 0.05 & -3.4 & active subgiant \\
\hline TYC 7318-593-1 & $15: 31: 21.93-33: 29: 39.5$ & 94 & 98 & G9.5V & 0.0 & 0.15 & -3.3 & active dwarf \\
\hline TYC 7858-526-1 & $16: 38: 38.47-39: 33: 03.5$ & 98 & 100 & F8.5IV & 2.5 & 0.02 & -4.0 & active subgiant $(\mathrm{CAB})$ \\
\hline HIP 63797 & $13: 04: 30.96-65: 55: 18.5$ & 9 & 73 & G3.5IV & 2.4 & 0.00 & $<-4.5$ & subgiant \\
\hline HIP 65423 & $13: 24: 35.12-55: 57: 24.2$ & 99 & 100 & GOV & 2.1 & 0.18 & -3.6 & $\mathrm{ZAMS}^{\mathrm{a}}$ \\
\hline HIP 68726 & 14:04:07.12 -37:15:50.5 & 60 & 93 & G0.5III & 1.9 & 0.00 & $<-5.1$ & giant \\
\hline HIP 72070 & $14: 44: 30.96-39: 59: 20.6$ & 62 & 91 & G1V & 2.6 & 0.16 & $<-4.2$ & ZAMS \\
\hline HIP 74501 & $15: 13: 29.22-55: 43: 54.6$ & 3 & 61 & G1.5III & 2.4 & 0.03 & $<-4.9$ & giant \\
\hline HIP 77015 & $15: 43: 29.86-38: 57: 38.6$ & 61 & 91 & $\mathrm{G} 0.5 \mathrm{~V}$ & 2.8 & 0.06 & $<-4.1$ & dwarf \\
\hline HIP 79610 & $16: 14: 43.02-38: 38: 43.5$ & 37 & 74 & $\mathrm{G} 0.5 \mathrm{~V}$ & 2.7 & 0.03 & $<-4.2$ & dwarf \\
\hline HIP 81775 & $16: 42: 10.36-31: 30: 15.0$ & 14 & 67 & G1IV & 2.8 & 0.04 & $<-4.1$ & subgiant \\
\hline
\end{tabular}

Note.- Columns: (1) Name from Tycho-2 or Hipparcos catalogs, (2) J2000.0 position, (3) Membership probability (with $v_{d i s p}=$ $1 \mathrm{~km} \mathrm{~s}^{-1}$ ), (4) Membership probability (with $v_{d i s p}=3 \mathrm{~km} \mathrm{~s}^{-1}$ ), (5) Spectral type (see §4.1), (6) Equivalent width of $\mathrm{H} \alpha \lambda 6562.8$ $(\AA),(7)$ Corrected EW of Li I $\lambda 6707.8(\AA),(8)$ Logarithm of ratio between X-ray and bolometric luminosities (approximate upper limits assume RASS PSPC detection limit of $0.05 \mathrm{ct} \mathrm{s}^{-1}$ and HR1 $=0$ ), (9) Class of object

${ }^{\mathrm{a}} \mathrm{HIP} 65423=\mathrm{HD}$ 116402. Cutispoto et al. (2002) measure $\mathrm{EW}(\mathrm{Li})=220 \mathrm{~m} \AA$, and $v \sin i=35 \mathrm{~km} \mathrm{~s}^{-1}$. 
TABLE 8

Age Estimates of LCC \& UCL

\begin{tabular}{ccccc}
\hline \hline \multicolumn{5}{c}{ Ref. Tracks method age(LCC) } \\
& & & age(UCL) \\
(Myr) & (Myr) \\
\hline 1 & 4 & pre-MS & $17 \pm 1$ & $15 \pm 1$ \\
1 & 5 & pre-MS & $21 \pm 2$ & $19 \pm 1$ \\
1 & 6 & pre-MS & $23 \pm 2$ & $22 \pm 1$ \\
\hline 1 & 7 & turn-off & $16 \pm 1$ & $17 \pm 1$ \\
\hline 2 & 8 & turn-off & $11-12$ & $14-15$ \\
3 & 8,9 & turn-off & $10-11$ & $12-13$ \\
\hline \hline
\end{tabular}

Note.-Uncertainties are standard errors of the mean. Pre-MS age estimates exclude known SBs and stars with $\log \mathrm{T}_{\text {eff }}<3.73$, which bias the calculated ages. The turn-off ages from this work are determined using only early-B stars classified as members by de Zeeuw et al. (1999).

REFERENCES.-(1) this work, (2) de Geus et al. (1989), (3) de Zeeuw \& Brand (1985), (4) D'Antona \& Mazzitelli (1997), (5) Palla \& Stahler (2001),

(6) Siess, Dufour, \& Forestini (2000),

(7) Bertelli et al. (1994), (8) Maeder

(1981), (9) Cogan (priv. comm.) 
TABLE 9

Revised Luminosity Classes of Standard Stars

\begin{tabular}{lccccc}
\hline \hline Star & $\begin{array}{c}\text { Published } \\
\text { Lum. class }\end{array}$ & $\begin{array}{c}\log g \\
\text { estimates }\end{array}$ & \multicolumn{2}{c}{$\begin{array}{c}\text { Sr/Fe HRD } \\
\text { class }\end{array}$} & $\begin{array}{c}\text { adopted } \\
\text { pos. }\end{array}$ \\
& Lum. class \\
\hline HR 5072 & V & $3.8-3.9$ (IV-V) & IV-V & IV-V & IV-V \\
HR 4995 & IV-V & $3.0-3.7$ (III/IV) & IV & IV & IV \\
HR 5409 & IV & $3.3-3.9$ & (III/IV) & III-IV III-IV & III-IV \\
HR 6608 & IIIb & $\ldots$ & IV & IV & IV \\
\hline \hline
\end{tabular}

Note.-Published luminosity classes from Keenan \& McNeil (1989). The range of published $\log g$ estimates come from the compilation of Cayrel de Strobel et al. (2001). "Sr/Fe class" is from measuring the Fe I $\lambda 4071 / \mathrm{Sr}$ II $\lambda 4071$ ratio in our spectra and intercomparison to the spectral standards in Table 3 (see Fig. 1). The luminosity class from the HRD position uses the $\mathrm{V}$ and $B-V$ data from it Hipparcos and the standard relations from Appendix B of Gray (1991). 